\title{
molecules
}

ISSN 1420-3049

www.mdpi.com/journal/molecules

Article

\section{Synthesis, Reactions and Antimicrobial Activities of 8-Ethoxycoumarin Derivatives}

\author{
Hany M. Mohamed ${ }^{1,3, *}$, Ashraf H. F. Abd El-Wahab ${ }^{2,3}$, Kamal A. Ahmed ${ }^{3}$, \\ Ahmed M. El-Agrody ${ }^{3,4}$, Ahmed H. Bedair ${ }^{3}$, Fathy A. Eid ${ }^{3}$ and Mostafa M. Khafagy ${ }^{3}$ \\ 1 Chemistry Department, Faculty of Medicine, Jazan University, Jazan 82621, Saudi Arabia \\ 2 Chemistry Department, Faculty of Science, Jazan University, Jazan 2097, Saudi Arabia \\ 3 Chemistry Department, Faculty of Science, Al-Azhar University, Nasr City, Cairo 11884, Egypt \\ 4 Chemistry Department, Faculty of Science, King Khalid University, Abha 9004, Saudi Arabia \\ * Author to whom correspondence should be addressed; E-Mail: hanysm83@yahoo.com; \\ Tel.: +966-50-762-5189.
}

Received: 14 November 2011; in revised form: 1 January 2012 / Accepted: 4 January 2012 / Published: 18 January 2012

\begin{abstract}
Condensation of 3-acetyl-8-ethoxycoumarin (3) with thiosemicarbazide gave ethylidenehydrazinecarbothioamide $\mathbf{5}$, which was transformed into the thiazolidin-4-one derivatives 6,7. Interaction of $\mathbf{3}$ with $\mathrm{DMF} / \mathrm{POCl}_{3}$ gave $\beta$-chloroacroline derivative $\mathbf{8}$. Treatment of $\mathbf{3}$ with malononitrile gave benzo[c]chromone and 2-aminobenzonitrile derivatives 9 and 10, respectively with respect to the reaction conditions. Condensation of 3-(2-bromoacetyl)-8-ethoxycoumarin (4) with $o$-phenylenediamine gave 3-(quioxaline-2yl)-8-ethoxycoumarin hydrobromide (11), while 4 reacted with 2-aminopyridine to give chromenopyridopyrimidine derivative 12. Condensation of 4 with potassium thio-cyanate/methanol gave an unexpected derivative, $2 \mathrm{H}$-chromeno-3-carboxy(methylcarbonimidic)thioanhydride 16, which upon treatment with $\left(\mathrm{NH}_{2}\right)_{2} \cdot \mathrm{H}_{2} \mathrm{O}$ gave 3-ethoxy-2-hydroxybenzaldehyde azine 19. Interaction of 4 with thiourea derivatives gave thiazole derivatives 20a-c. The structures of the newly synthesized compounds were confirmed by their spectra data. The newly synthesized compounds were also screened for their antimicrobial activity.
\end{abstract}

Keywords: 3-acetyl-8-ethoxycoumarin; bromination; active methylene; thiazole derivatives; thiosemicarbazide; KSCN; antimicrobial activities 


\section{Introduction}

Coumarin and its derivatives are used as additives in food, perfumes, cosmetics, pharmaceuticals, agrochemicals [1,2], for their spasmolytic, cardiothioc, antiviral, anticancer properties [3,4] and as laser dyes in the blue-green region. These types of dyes have been employed as labels for fluorescent energy transfer experiments [5,6]. Coumarin compounds also form a group of more than 40 drugs, which are widely used in medicine as anticoagulant, hypertensive, antiarrhythmic and immunomodulant agents [7]. Many coumarins were tested for various kinds of biological activity and their structures established based on chemical analytical techniques and spectroscopic methods [8-15]. The present study is a part of our programme [16-28] directed towards the synthesis of novel coumarin and chromene derivatives and evaluation of their antimicrobial activities.

\section{Results and Discussion}

\subsection{Chemistry}

Treatment of 3-ethoxysalicylaldehyde (1) with ethyl acetoacetate (2) in boiling ethanol containing few drops of piperidine afforded 3-acetyl-8-ethoxycoumarin (3) [29]. Bromination of 3 in acetic acid gave the corresponding $\omega$-bromo-8-ethoxy-3-acetylcoumarin (4) [29] (Scheme 1).

Scheme 1. Synthesis of 3-(2-bromoacetyl)-8-ethoxycoumarin (4).<smiles>CCOc1cccc(C=O)c1O</smiles>

1<smiles>CCOC(=O)CC(C)=O</smiles>

2<smiles>CCOc1cccc2cc(C(C)=O)c(=O)oc12</smiles>

3<smiles>CCOC(=O)c1cc2cccc(OCC)c2oc1=O</smiles>

4

Condensation of 3 with thiosemicarbazide afforded 2-[1-(8-ethoxycoumarin-3-yl)ethylidene]hydrazinecarbothioamide (5). Reaction of 5 with chloroacetic acid or ethyl chloroacetate afforded 2-[(1-(8-ethoxycoumarin-3-yl)ethylidene]hydrazonothiazolidin-4-one (6). Treatment of the thiazolidin-4one derivative 6 with $p$-methoxybenzaldehyde or $\alpha$-cyano- $p$-methoxycinnamonitrile in ethanolic piperidine afforded the corresponding 2-[(1-(8-ethoxycoumarin-3-yl)ethylidene)hydrazono]-5-(4methoxybenzylidene)thiazolidin-4-one (7) (Scheme 2).

Scheme 2. Synthesis hydrazone derivatives 5-7.

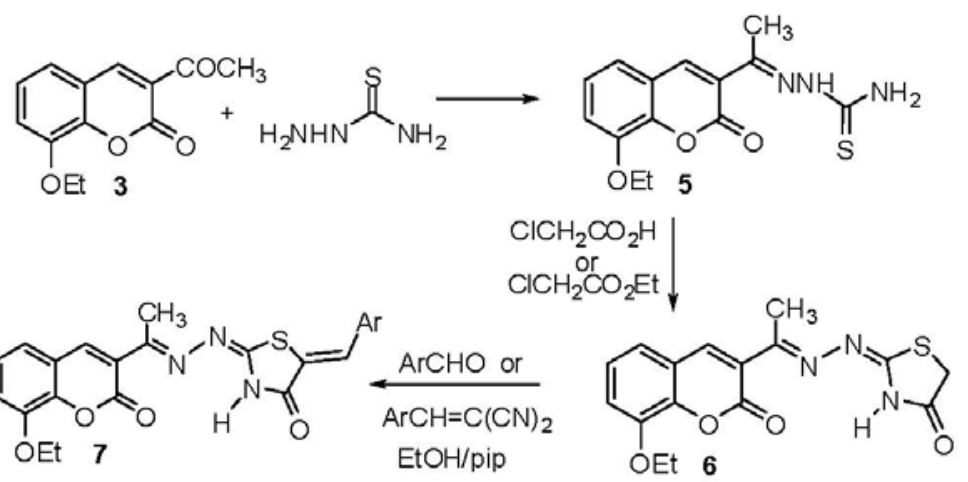

$\mathrm{Ar}=p-\mathrm{OCH}_{3} \mathrm{C}_{6} \mathrm{H}_{4}$ 
Structures 5-7 were established on the basis of spectral data. IR spectra showed the presence of $\mathrm{NH}_{2}$ and $\mathrm{NH}$ absorptions at $v 3,404,3,254,3,174 \mathrm{~cm}^{-1}$ and $\mathrm{CO}$ at $v 1,720 \mathrm{~cm}^{-1}$ for 5, NH at v 3,147 $\mathrm{cm}^{-1}, v 2,977 \mathrm{C}-\mathrm{H}$ (aliphatic), $\mathrm{CO}$ at $v 1,720,1,648 \mathrm{~cm}^{-1}$ for 6 and a $\mathrm{CO}$ at $v 1,705$, $1,655 \mathrm{~cm}^{-1}$ and $v 1,605 \mathrm{~cm}^{-1}$ for $\mathrm{N}=\mathrm{C}$ and $2,939.3 \mathrm{~cm}^{-1}$ for 7 . The ${ }^{1} \mathrm{H}-\mathrm{NMR}$ spectrum of 6 showed signals at $\delta 12.05 \mathrm{ppm}($ brs, $1 \mathrm{H}, \mathrm{NH}), 3.87 \mathrm{ppm}\left(\mathrm{s}, 2 \mathrm{H}, \mathrm{CH}_{2}\right), 2.49 \mathrm{ppm}\left(\mathrm{s}, 3 \mathrm{H}, \mathrm{CH}_{3}\right)$ and that of 7 signals at $\delta 12.40$ (brs, $1 \mathrm{H}, \mathrm{NH}), 8.44 \mathrm{ppm}(\mathrm{s}, 1 \mathrm{H}, 4-\mathrm{H}), 8.25 \mathrm{ppm}(\mathrm{s}, 1 \mathrm{H},=\mathrm{CH}-\mathrm{Ar}), 3.84 \mathrm{ppm}(\mathrm{s}, 3 \mathrm{H}$, $\mathrm{OCH}_{3}$ ). The mass spectra of compounds $\mathbf{5 , 6}$ provided additional evidence in support of the proposed structures.

Treatment of 8-ethoxy-3-acetylcoumarin (3) with $\mathrm{DMF} / \mathrm{POCl}_{3}$ gave 3-chloro-3-(8-ethoxycoumarin3-yl)acrylaldehyde (8) (Scheme 3). The formation of $\mathbf{8}$ indicates that the enolate form of $\mathbf{3}$ attacks the chloroiminium salt to gave the iminium ion which is hydrolyzed to the $\beta$-chloroacroline derivative 8 [30].

Scheme 3. Synthesis of $\beta$-chloroacroline derivative $\mathbf{8}$.
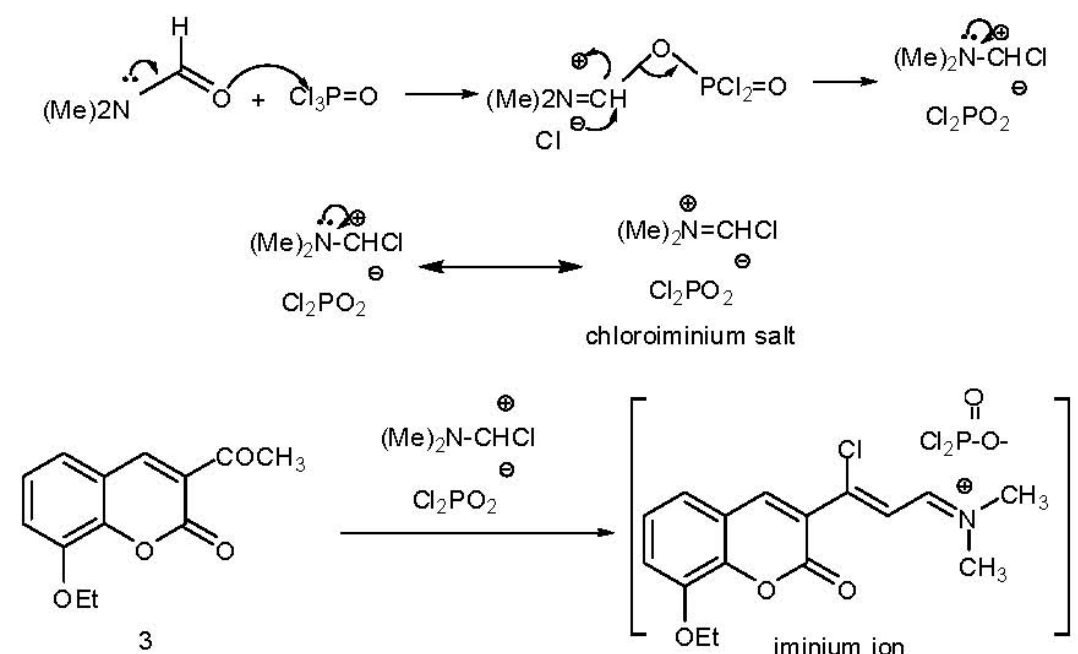<smiles>CCOc1cccc2cc(/C(Cl)=C/C=O)c(=O)oc12</smiles>

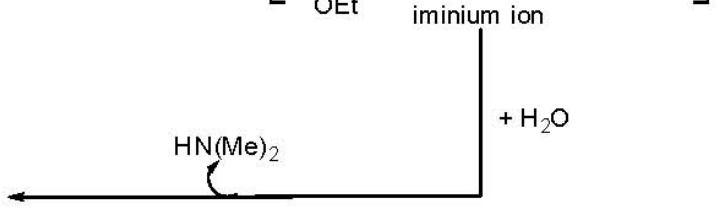

Interaction of 3 with malononitrile or ethyl cyanoacetate in ethanolic triethylamine solution gave the same product, the benzo[c]chromene-6-one derivative 9 (Scheme 4).

Scheme 4. Synthesis of benzo[c]chromene-6-one derivative 9.

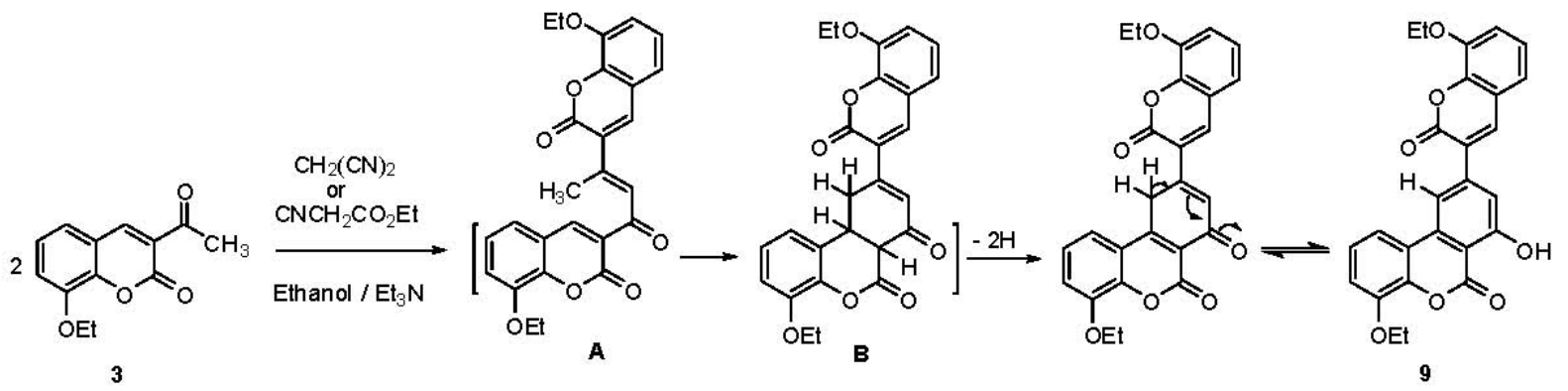


The formation of $\mathbf{9}$ can be explained by a self condensation of $\mathbf{3}$ to give a chalcone (A) as intermediate which undergoes an intramolecular cyclization through the addition of an active methylene group to the activated 3,4-double bond forming the intermediate $(\mathbf{B})$, which then underges spontaneous oxidation to the final product 9 [31] (Scheme 4).

Treatment of $\mathbf{3}$ with malononitrile in boiling methanolic piperidine solution instead of triethyl-amine, gave a product which formulated as 2-amino-4,6-bis(8-ethoxycouarin-3-yl)benzonitrile (10) (Scheme 5). The formation of $\mathbf{1 0}$ could be explained by condensation of the intermediate (A) with malononitrile to give another intermediate $(\mathbf{C})$ which underges intramolecular cyclization through the nucleophilc addition of active methylene group to one of the carbonitrile functions followed by aromatization [32] (Scheme 5).

Scheme 5. Synthesis of 2-amino-4,6-bis(8-ethoxycouarin-3-yl)benzonitrile (10).

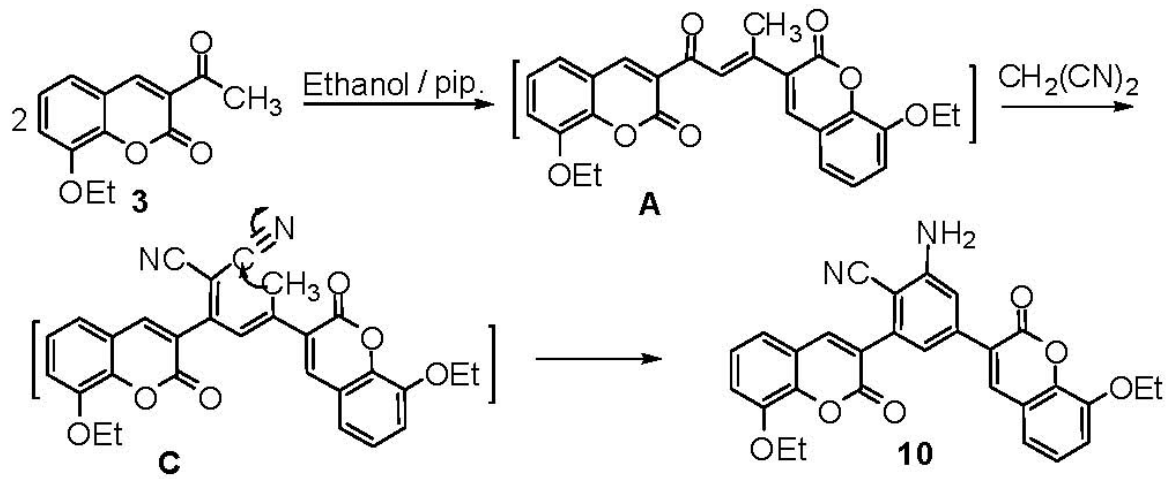

Treatment of 4 with an equimolar amount of $o$-phenylenediamine in boiling methanol resulted in the formation of 3-(quinoxalin-2-yl)-8-ethoxycoumarin hydrobromide (11) (Scheme 6). The formation of 11 [33] may be explained by cyclocondensation of $\omega$-bromo-8-ethoxy-3-acetylcoumarin (4) with $o$-phenylenediamine, followed by subsequent oxidation (Scheme 6).

Scheme 6. Synthesis of 3-(quinoxalin-2-yl)-8-ethoxycoumarin hydrobromide (11).
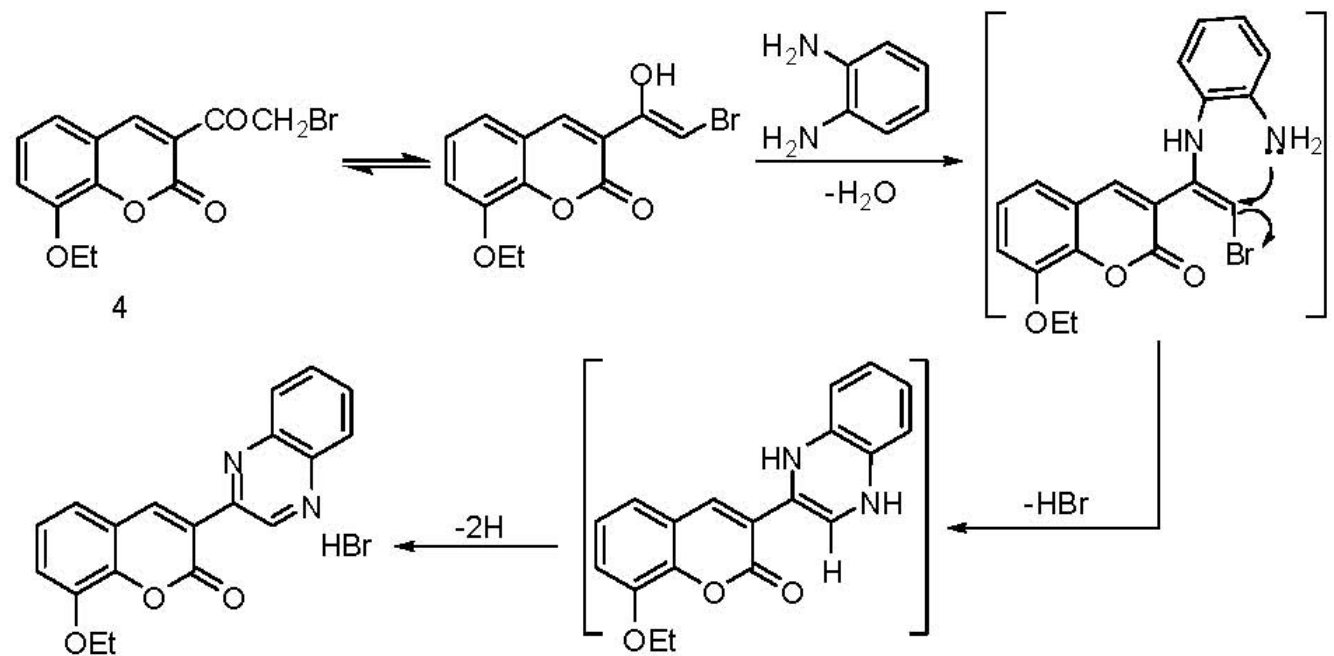

11

In a similar manner, 4 reacted with 2-aminopyridine to give the 4-ethoxychromeno[4,3$d]$ pyrido[1,2-a]pyrimidin-6-(7H)-one derivative 12 rather than the expected 3-imidazo[1,2-a]pyridine2-yl-8-ethoxychrome-2-one 13 [34] (Scheme 7). 
Scheme 7. Synthesis of chromenopyridopyrimidine 12.

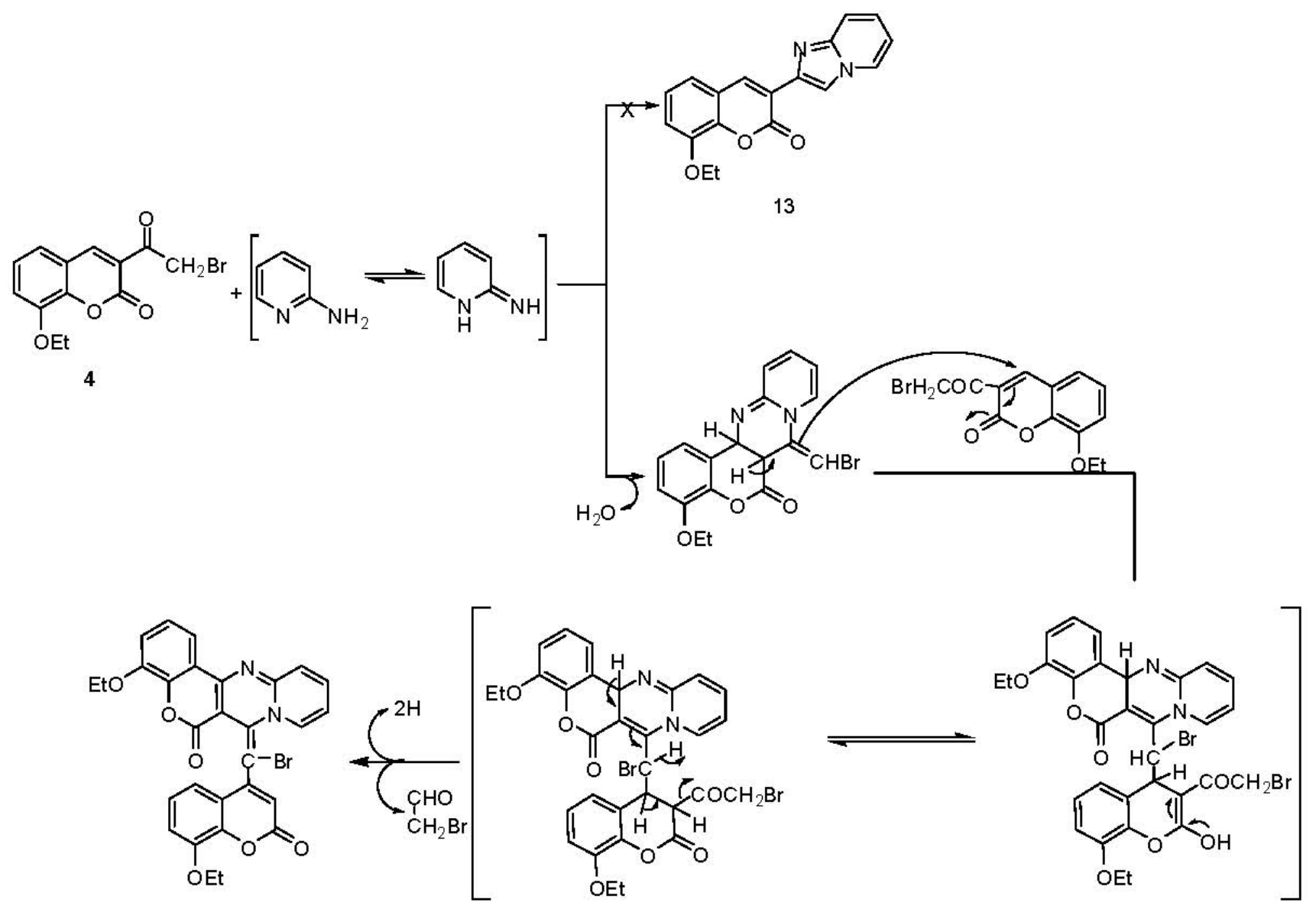

12

The formation of $\mathbf{1 2}$ can be explained by a nucleophilic addition of the more nucleophilic cyclic secondary nitrogen of 2-aminopyridine to the electrophilic carbonyl carbon of 3-(2-bromoacetyl)-8ethoxycoumarin (4) instead of nucleophilic replacement of bromine; the addition intermediate undergoes a subsequent cyclodehydration with the addition of the exonucleophilic nitrogen atom of 2-aminopyridine at position 2 to the active site of the coumarin ring (C-4), and the intermediate then acts as a nucleophile which attacks a second molecule of 4 at C-4 followed by aromatization and elimination of a bromoacetaldehyde molecule to give the final product 12. Previously Ramanna et al. [35] had stated that 3-( $\omega$-bromoacetyl)coumarins 14 on reaction with potassium thiocyanate gave 3-thiocyanatoacetylcoumarins 15 (Scheme 8).

Scheme 8. Synthesis of 3-thiocyanato derivatives 15.<smiles>[R]c1cc([R])c2oc(=O)c(C(=O)CBr)cc2c1</smiles>

14<smiles>[R]c1cc([R])c2oc(=O)c(C(=O)OCC3CCCC3)cc2c1</smiles> 
In the present research, treatment of 4 with $\mathrm{KSCN}$ in boiling methanol gave 2-bromoethylene-8ethoxy-2H-chromene-3-carboxylic(methylcarbonimidic)thioanhydride (16) rather than the corresponding 3-thiocyanatoacetylcoumarin derivative 17 (Scheme 9).

Scheme 9. Synthesis of compounds 16, 18 and 19 with MS fragmentaion patterns.

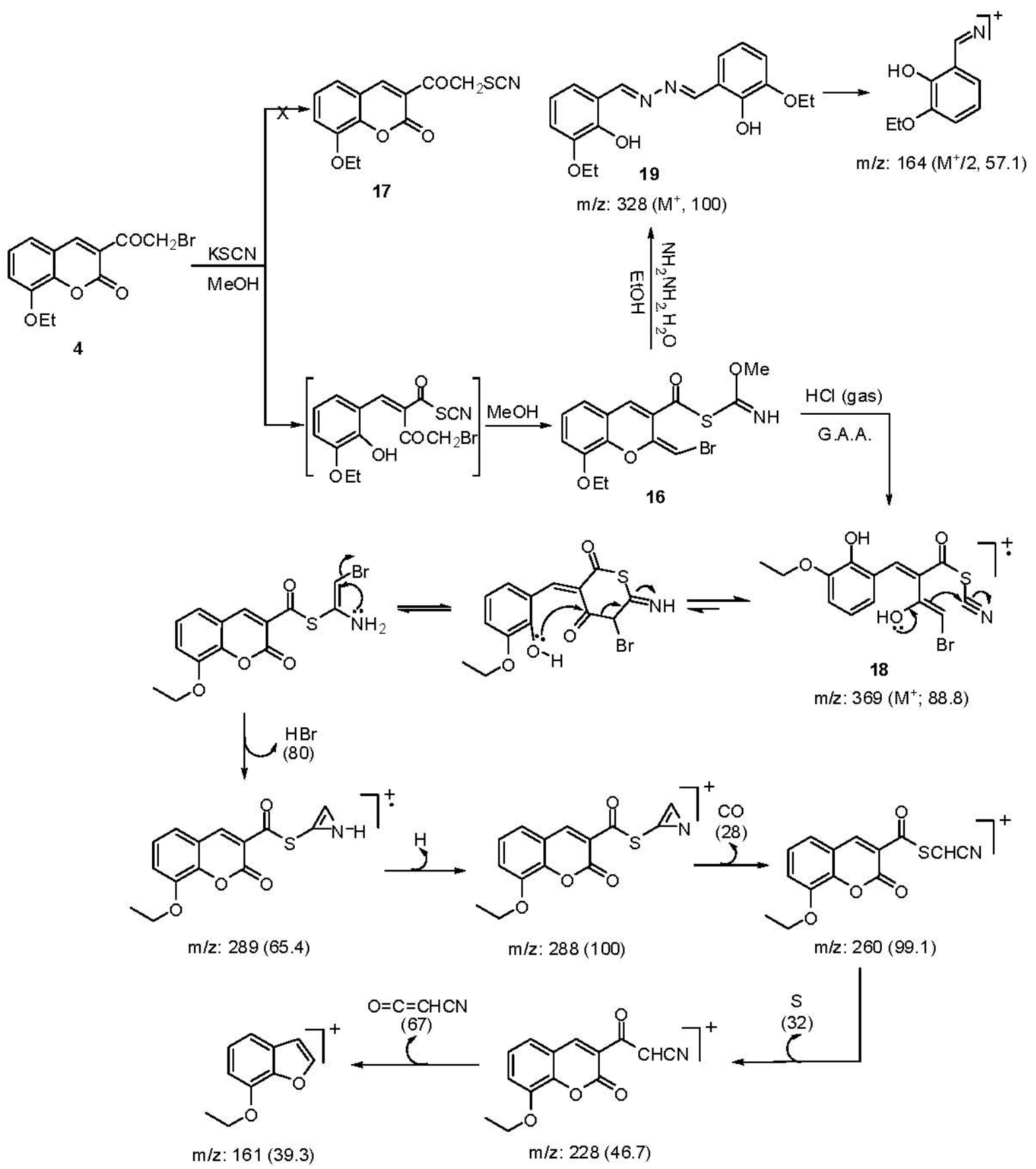

The structure was supported by the following evidence: (i) in the IR spectrum, the absence of SCN and the presence of $\mathrm{NH}$ at $3,141 \mathrm{~cm}^{-1}$; (ii) the ${ }^{1} \mathrm{H}-\mathrm{NMR}$ spectrum exhibited a characteristic singlet for $\mathrm{OCH}_{3}$ protons at $4.10 \mathrm{ppm}$ and at 7.88 as singlet signal for $=\mathrm{CH}-\mathrm{Br}$; (iii) the mass spectrum gave a molecular ion peak at $m / z(\%): 385\left(\mathrm{M}^{+}+2,37.3\right), 383\left(\mathrm{M}^{+}, 42.4\right)$ with a base peak at $303(\mathrm{M}-\mathrm{HBr}$, 100). The formation of $\mathbf{1 6}$ indicates that the strong nucleophile $\mathrm{SCN}^{-}$attacks the lactone carbonyl with concomitant addition of a methanol molecule and cyclization to give a chromene nucleus. When $\mathbf{1 6}$ 
was treated with $\mathrm{HCl}_{\text {gas }} / \mathrm{AcOH}$, a methanol molecule was eliminated and the chromene ring was opened to furnish 4-bromo-2-(3-ethoxy-2-hydroxybenzylidene)-3-hydroxybut-3-enoic cyanic thioanhydride (18) (Scheme 9). The mass spectrum of 18 gave a molecular ion peak at $\mathrm{m} / \mathrm{z}(\%): 369$ $(\mathrm{M}+, 88.8)$ together with a base peak at 288 (100), and other peaks at 289 (65.4), 260 (99.0), 228 (46.7), 161 (39.3). Interaction of 16 with ethanolic hydrazine hydrate solution effected ring opening with scission of the $\mathrm{C}_{3}-\mathrm{C}_{4}$ bond [36] and gave 3-ethoxy-2-hydroxybenzaldehyde azine (19) (Scheme 9). IR gave $v \mathrm{C}=\mathrm{N}$ at 1,562 and $1,597 \mathrm{~cm}^{-1}$, and $\mathrm{OH}$ at $3,302 \mathrm{~cm}^{-1},{ }^{1} \mathrm{H}-\mathrm{NMR}$ ppm: 9.20 $(\mathrm{s}, 1 \mathrm{H}, \mathrm{CH}=\mathrm{N}), 12.10,12.45$ (phenolic $\mathrm{OH}$ ). Its mass spectrum gave a molecular ion peak at $\mathrm{m} / \mathrm{z}$ (intensity \%): 328 (100) as a base peak, and other peaks at 164 (M/2, 57.12), 136 (45.7), 121 (50.9), 80 (65.3).

Interaction of 4 with thiourea, phenylthiourea and cyanothioacetamide in boiling methanol afforded the thiazole derivatives 20a-c [37-39] respectively (Scheme 10). Interaction of 20c with neat triethyl orthoformate under reflux give the ethoxymethyleneamino derivative 21, which reacted with dimethylamine to give $N, N$-dimethylaminoethyleneamino derivative 22 (Scheme 10).

Scheme 10. Synthesis of coumarinothiazole derivatives 20, 21 and 22.

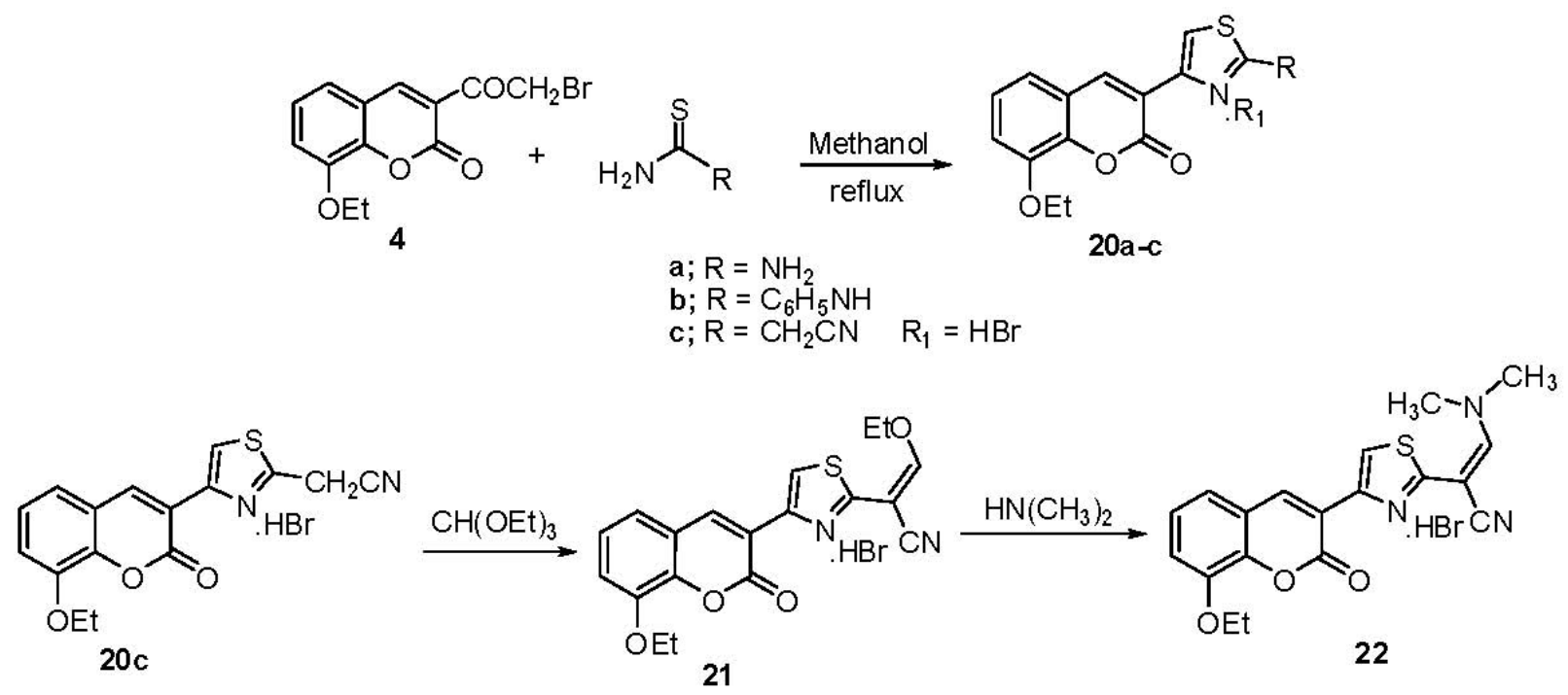

The structures of 20-22 were confirmed by their spectral data. The IR spectra showed $\mathrm{NH}_{2}$ bands at v 3,387, 3,302, 3,148 $\mathrm{cm}^{-1}$ and a CO band at $v 1,705 \mathrm{~cm}^{-1}$ for $\mathbf{2 0 a}$; $\mathrm{NH}$ bands at $v 3,302,3,148 \mathrm{~cm}^{-1}$ and $\mathrm{CO}$ band at $v 1,697 \mathrm{~cm}^{-1}$ for $\mathbf{2 0 b}$, and $\mathrm{CN}$ band at $v 2,262 \mathrm{~cm}^{-1}$ and $\mathrm{CO}$ band at $v 1,722 \mathrm{~cm}^{-1}$ for 20c; C-H (aliphatic) at v 2,978 $\mathrm{cm}^{-1}, \mathrm{CN}$ band at $v 2,222 \mathrm{~cm}^{-1}$ a CO band at $v 1,728 \mathrm{~cm}^{-1}$ for 21 and a

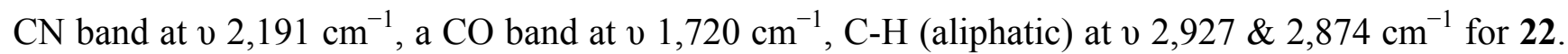
Characteristic ${ }^{1} \mathrm{H}-\mathrm{NMR}$ resonances were observed at $\delta 10.31(\mathrm{~s}, 1 \mathrm{H}, \mathrm{HNPh})$ and $8.61 \mathrm{ppm}(\mathrm{s}, 1 \mathrm{H}, \mathrm{H}-4)$ for 20b, $\delta 8.42(\mathrm{~s}, 1 \mathrm{H}, 4-\mathrm{H})$ and $4.55 \mathrm{ppm}\left(\mathrm{s}, 2 \mathrm{H}, \mathrm{CH}_{2}\right) 8.71(\mathrm{~s}, 1 \mathrm{H}$, thiazole-H) for 20c, $\delta 8.43(\mathrm{~s}, 1 \mathrm{H}$, 4-H) $8.15(\mathrm{~s}, 1 \mathrm{H},=\mathrm{CHOEt}), 8.70(\mathrm{~s}, 1 \mathrm{H}$, thiazole-H) for 21 and $8.12 \mathrm{ppm}(\mathrm{s}, 1 \mathrm{H}, 4-\mathrm{H})$ and $3.28 \mathrm{ppm}$ $\left(\mathrm{s}, 6 \mathrm{H}, \mathrm{N}\left(\mathrm{CH}_{3}\right)_{2}\right), 7.89\left[\mathrm{~s}, 1 \mathrm{H},=\mathrm{CHN}(\mathrm{Me})_{2}\right], 8.74(\mathrm{~s}, 1 \mathrm{H}$, thiazole-H) for 22. The mass spectrum of 20c gave a molecular ion peak at $m / z(\%) 394\left(\mathrm{M}^{+}+2,24.90\right)$ and $392\left(\mathrm{M}^{+}, 28.90\right)$ with a base peak at 284 $\left(\mathrm{M}^{+}-(\mathrm{HBr}+\mathrm{CO}), 100\right)$; for 21: $\mathrm{m} / z(\%) 448\left(\mathrm{M}^{+}, 12.1\right), 450\left(\mathrm{M}^{+}+2,10.1\right)$ and a base peak at 368 (M-HBr, 100); for 22: $m / z$ : $449\left(\mathrm{M}^{+}+2,14.1\right), 447\left(\mathrm{M}^{+}, 18\right), 367\left(\mathrm{M}^{+}-\mathrm{HBr}, 100\right)$. 
Condensation of 20c with various aromatic aldehydes 23 afforded the corresponding dicoumarin-3-ylthiazole derivatives 24, 25 and iminocoumarin derivative 26, while condensation of 20c with aromatic aldehydes in methanolic piperidine solution give 27a,b. Compound 20c was also readily coupled with $p$-methoxybenzenediazonium chloride to afford $\mathbf{2 8}$ (Scheme 11). The structure of $\mathbf{2 8}$ was supported by its independent synthesis from 4 and (4-methoxyphenylazo)-2cyanoethanethioamide (29) (Scheme 11).

Scheme 11. Synthesis of coumarin-3-ylthiazole derivatives 24-28.

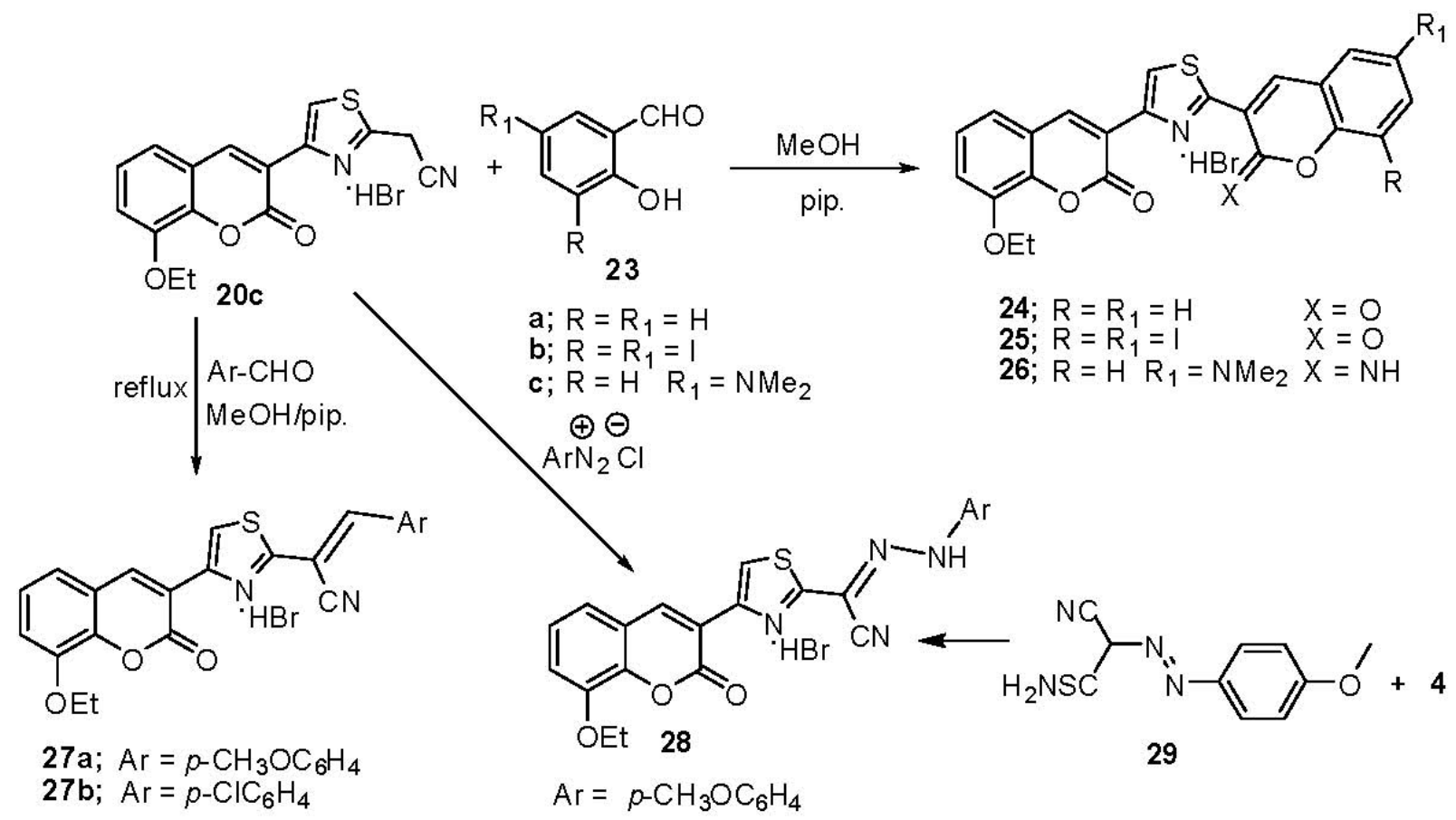

Structures 24-28 were established on the basis of spectral data. The IR spectra showed the presence of a CO bands at $v 1,720 \mathrm{~cm}^{-1}$ for 24-26, while a NH band appeared at $v 3,449,3,209 \mathrm{~cm}^{-1}$ for 26; a $\mathrm{CN}$ and at $v 2,207 \mathrm{~cm}^{-1}$ a CO band at $v 1,728 \mathrm{~cm}^{-1}$ for $27 \mathbf{a}$; a CN band at $v 2,214 \mathrm{~cm}^{-1}$ and a $\mathrm{CO}$ band at $v 1,720 \mathrm{~cm}^{-1}$ for $\mathbf{2 7 b}$ and a $\mathrm{NH}$ band at $v 3,156 \mathrm{~cm}^{-1}$ a CN band at $v 2,214 \mathrm{~cm}^{-1} \mathrm{CO}$ at $v 1,736 \mathrm{~cm}^{-1}$ for 28. The ${ }^{1} \mathrm{H}-\mathrm{NMR}$ spectra showed two signals at $\delta 8.95,8.54 \mathrm{ppm}(2 \mathrm{~s}, 2 \mathrm{H}, \mathrm{H}-4) 9.14(\mathrm{~s}, 1 \mathrm{H}$, thiazole-H) for 24; at $\delta 8.39$ (s, 2H, 2H-4) and $3.31 \mathrm{ppm}\left(\mathrm{s}, 6 \mathrm{H}, 2 \mathrm{CH}_{3}\right)$ for 26 and $\delta 11.93(\mathrm{~s}, 1 \mathrm{H}, \mathrm{NH}$, exchangeable with $\left.\mathrm{D}_{2} \mathrm{O}\right), 8.39(\mathrm{~s}, 1 \mathrm{H}, \mathrm{H}-4)$ and $3.76 \mathrm{ppm}\left(\mathrm{s}, 3 \mathrm{H}, \mathrm{OCH}_{3}\right)$ for $\mathbf{2 8}$. The mass spectra of compounds 24-28 provided additional evidence for the proposed structures.

\section{Experimental}

\subsection{General}

Melting points were determined with a Stuart Scientific Co. Ltd. apparatus. IR spectra were determined as $\mathrm{KBr}$ pellets on a Jasco FT/IR 5300 spectrophotometer. ${ }^{1} \mathrm{H}-\mathrm{NMR}$ spectra were recorded using a Varian Mercury (300 MHz) spectrometer. The mass spectra recorded on a Shimadzu GC-MS QP 1000 EX spectrometer. Elemental analyses were performed on a Perkin-Elmer 240 microanalyser in the Cairo University Faculty of Science. 
Synthesis of 3-acetyl-8-ethoxycoumarin (3). 3-Ethoxysalicylaldehyde 1 (10 mmol) was refluxed with ethyl acetoacetate $2(10 \mathrm{mmol})$ in ethanolic/piperidine solution $(20 \mathrm{~mL}, 0.5 \mathrm{~mL})$ for two hours. The precipitate formed on cooling was collected by filtration, washed with cold ethanol and dried. The solid formed was recrystallized from diluted ethanol to give pale yellow crystals; yield 88\%; m.p. 130-132 ${ }^{\circ} \mathrm{C}$, lit. $\left(135-7^{\circ} \mathrm{C}\right)$ [29]; IR (KBr) v $\left(\mathrm{cm}^{-1}\right)$ : 3,060, 2,976, 2,874 (CH stretching), 1,730, 1,678 (CO); ${ }^{1} \mathrm{H}-\mathrm{NMR}\left(\mathrm{DMSO}_{\mathrm{d}}\right) \delta: 1.40\left(\mathrm{t}, 3 \mathrm{H}, \mathrm{CH}_{3}, J=7.2 \mathrm{~Hz}\right), 2.58$ (s, 3H, $\left.\mathrm{COCH}_{3}\right), 4.19$ (q, 2H, $\mathrm{CH}_{2}$, $J=7.2 \mathrm{~Hz}$ ), 7.31-7.65 (m, 3H, Ar-H), 8.59 (s, 1H, H-4); ${ }^{13} \mathrm{C}-\mathrm{NMR}$ (DMSO-d 6 ) $\delta: 13.90$ ( $\mathrm{CH}_{3}$-ester), $25.72\left(\mathrm{CH}_{3}\right.$-acetyl), 62.33 ( $\mathrm{CH}_{2}$-ester), 115.40 (C-7), 118.21 (C-5), 120.11 (C-4a), 126.37 (C-6), 128.00 (C-3), 145.03 (C-4), 150.80 (C-8a), 154.12 (C-8), 160.04 (CO-lactone), 175.19 (CO-acetyl); Anal. calcd. for $\mathrm{C}_{13} \mathrm{H}_{12} \mathrm{O}_{4}$ : C, 67.22; $\mathrm{H}$ 5.17; found: C, 67.20; H, 5.16\%.

Synthesis of 3-(2-bromoacetyl)-8-ethoxycoumarin (4). A solution of 3 (10 mmol) in acetic acid $(10 \mathrm{~mL})$ was stirred with bromine $(0.50 \mathrm{~mL}, 10 \mathrm{mmol})$ for 2 hours in direct sun-light. The solid formed was collected by filtration, washed with acetic acid, then ethanol, and dried. The product was recrystallized from ethanol to give yellow crystals; yield: 79\%; m.p. $180-182{ }^{\circ} \mathrm{C}$; IR $(\mathrm{KBr}) v\left(\mathrm{~cm}^{-1}\right)$ : 3,080, 2,972, 2,952, (CH stretching), 1,722, 1,692 (CO); ${ }^{1} \mathrm{H}-\mathrm{NMR}\left(\mathrm{CDCl}_{3}\right)$ : 1.54 (t, 3H, $\mathrm{CH}_{3}$, $J=7.2 \mathrm{~Hz}), 4.23\left(\mathrm{q}, 2 \mathrm{H}, \mathrm{CH}_{2}, J=7.2 \mathrm{~Hz}\right), 4.77\left(\mathrm{~s}, 2 \mathrm{H}, \mathrm{CH}_{2}\right), 7.19-7.32(\mathrm{~m}, 3 \mathrm{H}, \mathrm{Ar}-\mathrm{H}), 8.60(\mathrm{~s}, 1 \mathrm{H}$, $\mathrm{H}-4) ;{ }^{13} \mathrm{C}-\mathrm{NMR}\left(\mathrm{DMSO}_{6}\right)$ \%: $13.73\left(\mathrm{CH}_{3}\right.$-ester), $35.10\left(\mathrm{CH}_{2} \mathrm{Br}\right) 62.13\left(\mathrm{CH}_{2}\right.$-ester), $115.47(\mathrm{C}-7)$, 118.43 (C-5), 120.01 (C-4a), 125.75 (C-6), 127.92 (C-3), 145.53 (C-4), 151.42 (C-8a), 154.19 (C-8), 162.70 (CO-lactone), 173.03 (CO-acetyl); $\mathrm{MS} \mathrm{m} / z$ (\%): $312\left(\mathrm{M}^{+}+2,29.02\right), 310\left(\mathrm{M}^{+}, 29.29\right)$, 231 (70.11), 217 (58.15), 203 (61.9), 189 (100); Anal. calcd. for $\mathrm{C}_{13} \mathrm{H}_{11} \mathrm{BrO}_{4}$ : C, 50.32; $\mathrm{H}$ 3.55; found: C, 50.33; H, 3.57\%.

Synthesis of 2-(1-(8-ethoxycoumarin-3-yl)ethylidene)hydrazinecarbothioamide (5). A solution of $\mathbf{3}$ $(10 \mathrm{mmol})$ in DMF $(10 \mathrm{~mL})$ was refluxed with thiosemicarbazide $(10 \mathrm{mmol})$ for 2 hours. The product formed was filtered off, washed with ethanol, dried and recrystallized from ethanol to give $\mathbf{5}$ as colourless needles; yield 81\%; m.p. 218-220 ${ }^{\circ} \mathrm{C}$; IR $(\mathrm{KBr}) v\left(\mathrm{~cm}^{-1}\right): 3,404,3,254,3,174$, (NH and $\mathrm{NH}_{2}$ ), 2,976 (C-H aliphatic), 1,720 (CO); MS m/z (\%): 305 (M+, 42), 290 (100), 245 (23), 230 (27), 161 (24); Anal. calcd. for $\mathrm{C}_{14} \mathrm{H}_{15} \mathrm{~N}_{3} \mathrm{O}_{3} \mathrm{~S}$ : C, 55.07; H, 4.95; N, 13.76; found: C, 55.09; H, 4.94; $\mathrm{N}, 13.78 \%$.

Synthesis of 2-((1-(8-ethoxycoumarin-3-yl)ethylidene)hydrazono)thiazolidin-4-one (6). A mixture of 5 $(10 \mathrm{mmol})$ and chloroacetic acid or ethyl chloroacetate $(10 \mathrm{mmol})$ was refluxed in acetic acid $(20 \mathrm{~mL})$ for 3 hours. The solid product was filtered off, washed with excess ethanol and recrystallized from acetic acid to give pale yellow crystals; yield 83\%; m.p. $238-240{ }^{\circ} \mathrm{C}$; IR (KBr) v $\left(\mathrm{cm}^{-1}\right): 3,147(\mathrm{NH})$, 2,977, (CH aliphatic), 1,720, 1,648 (CO); ${ }^{1} \mathrm{H}-\mathrm{NMR}\left(\mathrm{DMSO}_{-} \mathrm{d}_{6}\right) \delta: 1.41$ (t, 3H, $\left.\mathrm{CH}_{3}, J=6.9 \mathrm{~Hz}\right), 2.49$ (s, 3H, $\left.\mathrm{CH}_{3}\right), 3.83$ (s, 3H, $\left.\mathrm{OCH}_{3}\right), 3.87$ (s, 2H, $\left.\mathrm{CH}_{2}\right), 4.18$ (q, 2H, $\left.\mathrm{CH}_{2}, J=6.9 \mathrm{~Hz}\right), 7.29-7.36(\mathrm{~m}, 3 \mathrm{H}$, Ar-H), 8.46 (s, 1H, H-4), 12.05 (brs, 1H, NH); MS m/z (\%): 345 (M $\left.\mathrm{M}^{+}, 100\right), 344$ (94), 330 (58), 301 (53), 288 (61), 188 (55), 185 (51), 130 (50), 87 (59); Anal. calcd. For $\mathrm{C}_{16} \mathrm{H}_{15} \mathrm{~N}_{3} \mathrm{O}_{4} \mathrm{~S}$ : C, 55.64; $\mathrm{H}, 4.38$; N, 12.17; found: C, 55.66; H, 4.37; N, 12.19\%.

Synthesis of 2-((1-(8-ethoxycoumarin-3-yl)ethylidene)hydrazono)-5-(4-methoxybenz-ylidene)thiazolidin-4one (7). A solution of 6 (5 mmol), $p$-methoxybenzaldehyde or $\alpha$-cyano- $p$-methoxycinnamonitrile 
$(5 \mathrm{mmol})$ in ethanol $(30 \mathrm{~mL})$ containing few drops of piperidine was heated under reflux for 3 hours. The solid obtained on cooling was filtered off, washed with ethanol, dried and recrystallized from acetic acid to give pale brown crystals; yield 92\%; m.p. $220-2{ }^{\circ} \mathrm{C}$; IR $(\mathrm{KBr}) v\left(\mathrm{~cm}^{-1}\right): 2,939.3(\mathrm{CH}$ aliphatic), 1,705, 1,655 (CO), 1,605 ( $\mathrm{N}=\mathrm{C}) ;{ }^{1} \mathrm{H}-\mathrm{NMR}\left(\mathrm{DMSO}_{6}\right) \delta$ : $1.42\left(\mathrm{t}, 3 \mathrm{H}, \mathrm{CH}_{3}, J=6.9 \mathrm{~Hz}\right.$ ), $2.38\left(\mathrm{~s}, 3 \mathrm{H}, \mathrm{CH}_{3}\right), 3.83$ (s, 3H, $\left.\mathrm{OCH}_{3}\right), 4.20$ (q, 2H, $\left.\mathrm{CH}_{2}, J=6.9 \mathrm{~Hz}\right), 7.56-7.79$ (m, 7H, Ar-H), 8.25 (s, $1 \mathrm{H},=\mathrm{CH}-\mathrm{Ar}$ ), 8.44 (s, $1 \mathrm{H}, 4-\mathrm{H}), 12.40$ (brs, $1 \mathrm{H}, \mathrm{NH}$ ); Anal. calcd. for $\mathrm{C}_{24} \mathrm{H}_{21} \mathrm{~N}_{3} \mathrm{O}_{5} \mathrm{~S}$ : C, 62.19; $\mathrm{H}, 4.57$; N, 9.07; found: C, 62.18; H, 4.55; N, 9.09\%.

Synthesis of 3-chloro-3-(8-ethoxycoumarin-3-yl)acrylaldehyde (8). A solution of compound 3 (10 mmol) in DMF $(10 \mathrm{~mL})$ was cooled in an ice bath, a 1:1 mixture of DMF $(10 \mathrm{mmol})$ and (10 mmol) $\mathrm{POCl}_{3}$ was added to it dropwise while the temperature was kept at $0{ }^{\circ} \mathrm{C}$. The resulting mixture was stirred for 2 hours at $0{ }^{\circ} \mathrm{C}$, hen it was allowed to stir at room temperature for 3 hours more and poured onto crushed ice. The solid formed was filtered, washed with water and dried under vacuum. The crude product was recrystallized from ethanol to give pale yellow needles; yield 94\%; m.p. $123-125{ }^{\circ} \mathrm{C}$; IR (KBr) v $\left(\mathrm{cm}^{-1}\right)$ : 3,094, 2,978, 2,928 (CH stretching), 1,720, 1,668 (CO); ${ }^{1} \mathrm{H}-\mathrm{NMR}\left(\mathrm{DMSO}_{6}\right.$ ) $\delta: 1.42$ (t, 3H, $\left.\mathrm{CH}_{3}, J=7.2 \mathrm{~Hz}\right), 4.21\left(\mathrm{q}, 2 \mathrm{H}, \mathrm{CH}_{2}, J=7.2 \mathrm{~Hz}\right), 7.41(\mathrm{~d}, 1 \mathrm{H}$, vinyl proton, $J=6.9 \mathrm{~Hz}), 7.33-7.53(\mathrm{~m}, 3 \mathrm{H}, \mathrm{Ar}-\mathrm{H}), 8.81(\mathrm{~s}, 1 \mathrm{H}, \mathrm{H}-4), 10.19$ (d, 1H, CHO, $J=6.9 \mathrm{~Hz})$; MS m/z (\%): $280\left(\mathrm{M}^{+}+2,3.1\right), 278\left(\mathrm{M}^{+}, 6.4\right), 252$ (3), 250 (9), 224 (6), 222 (20), 187 (100); Anal. calcd. for $\mathrm{C}_{14} \mathrm{H}_{11} \mathrm{ClO}_{4}$ : C, 60.34; $\mathrm{H}, 3.98$; found: C, 60.43; H, 3.96\%.

Synthesis of 4-ethoxy-9-(8-ethoxycoumarin-3-yl)-7-hydroxy-6H-benzo[c]chromen-6-one (9). Method A: A solution of $1(10 \mathrm{mmol})$, malononitrile $(10 \mathrm{mmol})$ in absolute ethanol $(30 \mathrm{~mL})$ was refluxed in the presence of TEA $(0.2 \mathrm{~mL})$. A solid formed after $30 \mathrm{~min}$. boiling and the refluxing were continued for 1.5 hours more. The crude product was recrystallized from an ethanol/benzene mixture to give colourless crystals; yield 77\%; m.p. $236-8{ }^{\circ} \mathrm{C}$; IR $(\mathrm{KBr}) v\left(\mathrm{~cm}^{-1}\right): 3,425(\mathrm{OH}), 3,094,2,978,2,928$ (CH stretching), 1,704, 1,694 (CO); ${ }^{1} \mathrm{H}-\mathrm{NMR}\left(\mathrm{DMSO}_{6} \mathrm{~d}_{6}\right) \delta: 1.45$ (t, 3H, $\mathrm{CH}_{3}, J=7.0 \mathrm{~Hz}$ ), 4.20 (q, 2H, $\left.\mathrm{CH}_{2}, J=7.0 \mathrm{~Hz}\right), 7.30-8.16(\mathrm{~m}, 8 \mathrm{H}, \mathrm{Ar}-\mathrm{H}), 8.55(\mathrm{~s}, 1 \mathrm{H}, \mathrm{H}-4)$ and $11.22(\mathrm{~s}, 1 \mathrm{H}$, enolic $\mathrm{OH}$, exchangeable by $\left.\mathrm{D}_{2} \mathrm{O}\right)$; MS m/z (\%): $444\left(\mathrm{M}^{+}, 68\right), 388$ (100), 360 (28), 304 (21), 189 (15); Anal. calcd. for $\mathrm{C}_{26} \mathrm{H}_{20} \mathrm{O}_{7}$ : C, 70.25; H, 4.50. Found: C, 70.27; H, 4.52\%. Method B: The reaction was carried out as described in Method A, but using ethyl cyanoacetate $(1.13 \mathrm{~mL})$ instead of malononitrile; yield (82\%) (identity confirmed by m.p. and mixed m.p.).

Synthesis of 2-amino-4,6-bis(8-ethoxycoumarin-3-yl)benzonitrile (10). The reaction was carried out with the same procedure described for compound 9 (Method A) but using absolute methanol as a solvent and piperidine as a catalyst; m.p. $330-332{ }^{\circ} \mathrm{C}, 75 \%$; IR $(\mathrm{KBr}) v\left(\mathrm{~cm}^{-1}\right): 3,474,3,320\left(\mathrm{NH}_{2}\right)$, 3,092, 3,042, 2,980 (CH stretching); 2,206 (CN), 1,720, 1,696 (CO); ${ }^{1} \mathrm{H}-\mathrm{NMR}\left(\mathrm{DMSO}-\mathrm{d}_{6}\right) \delta: 1.43$ (t, $\left.3 \mathrm{H}, \mathrm{CH}_{3}, J=7.2 \mathrm{~Hz}\right), 4.23\left(\mathrm{q}, 2 \mathrm{H}, \mathrm{CH}_{2}, J=7.2 \mathrm{~Hz}\right), 7.31\left(\mathrm{~m}, 10 \mathrm{H}, \mathrm{Ar}-\mathrm{H}+\mathrm{NH}_{2}\right), 7.69(\mathrm{~s}, 1 \mathrm{H}, \mathrm{H}-4)$, 8.37 (s, 1H, H-4); MS m/z (\%): 494 ( $\left.\mathrm{M}^{+}, 27\right), 412$ (100), 384 (19), 344 (28), 316 (44), 243 (19), 242 (10), 206 (22); Anal. calcd. for $\mathrm{C}_{29} \mathrm{H}_{22} \mathrm{~N}_{2} \mathrm{O}_{6}$ : C, 70.44; H, 4.48; N, 5.67; found: C, 70.40; H, 4.47; N, 5.68\%.

Synthesis of 3-(quinoxalin-2-yl)-8-ethoxycoumarin hydrobromide (11). A solution of 4 (5 mmol) and $o$-phenylenediamine $(5 \mathrm{mmol})$ in absolute methanol $(20 \mathrm{~mL})$ was refluxed for 3 hours. The solid 
obtained was filtered, washed with ethanol and dried under vacuum. The crude product was recrystallized from ethanol/benzene mixture to give compound $\mathbf{1 1}$ as pale brown crystals; yield 77\%; m.p. 208-209 ${ }^{\circ} \mathrm{C}$; IR (KBr) v $\left(\mathrm{cm}^{-1}\right)$ : 3,417 broad (NH), 3,098, 3,022, 2,987, 2,896 (CH stretching); 1,720 (CO); ${ }^{1} \mathrm{H}-\mathrm{NMR}\left(\mathrm{DMSO}_{6}\right) \delta: 1.43\left(\mathrm{t}, 3 \mathrm{H}, \mathrm{CH}_{3}, J=7.1 \mathrm{~Hz}\right), 4.21$ (q, 2H, $\mathrm{CH}_{2}, J=7.1 \mathrm{~Hz}$ ), 7.37-8.15 (m, 8H, Ar-H), 8.88 (s, $1 \mathrm{H}, \mathrm{H}-4), 9.58$ (brs, $1 \mathrm{H}, \mathrm{NH}$, exchangeable by $\left.\mathrm{D}_{2} \mathrm{O}\right)$; $\mathrm{MS} \mathrm{m} / z(\%)$ : $400\left(\mathrm{M}^{+}+2,75\right), 398\left(\mathrm{M}^{+}, 76\right), 370$ (98), 368 (100), 289 (69), 205 (96), 102 (53), 76 (57); Anal. calcd. for $\mathrm{C}_{19} \mathrm{H}_{15} \mathrm{BrN}_{2} \mathrm{O}_{3}$ : C, 57.16; H, 3.79; N, 7.02; found: $\mathrm{C}, 57.26 ; \mathrm{H}, 3.69 ; \mathrm{N}, 6.98 \%$.

\section{7-(Bromo-(8-ethoxycoumarin-4-yl)methylene)-4-ethoxychromeno[4,3-d]pyrido[1,2-a]-pyrimidin-6(7H)-}

one (12). Compound 12 was prepared from 4 (5 mmol) and 2-aminopyridine $(5 \mathrm{mmol})$ according to the procedure described for $\mathbf{1 1}$ to give $\mathbf{1 2}$ from acetic acid as pale brown crystals; yield 63\%; m.p. 231-233 ${ }^{\circ} \mathrm{C}$; IR (KBr) v ( $\left.\mathrm{cm}^{-1}\right):$ 2,924 (CH stretching), 1,713, 1,692 (CO); ${ }^{1} \mathrm{H}-\mathrm{NMR}$ (DMSO-d ${ }_{6}$ ) $\delta: 1.18\left(\mathrm{t}, 3 \mathrm{H}, \mathrm{CH}_{3}, J=7.1 \mathrm{~Hz}\right), 1.37\left(\mathrm{t}, 3 \mathrm{H}, \mathrm{CH}_{3}, J=6.9 \mathrm{~Hz}\right), 4.02$ (q, 2H, $\left.\mathrm{CH}_{2}, J=7.1 \mathrm{~Hz}\right), 4.18$ (q, $\left.2 \mathrm{H}, \mathrm{CH}_{2}, J=6.9 \mathrm{~Hz}\right), 6.46(\mathrm{~s}, 1 \mathrm{H}, \mathrm{H}-3$, coumarin), 7.04-8.36 (m, 10H, Ar-H, pyridine-H); MS $\mathrm{m} / \mathrm{z}$ (\%): $574\left(\mathrm{M}^{+}+2,25\right), 572\left(\mathrm{M}^{+}, 31\right), 493$ (100), 465 (97), 304 (49), 280 (30), 224 (51), 196 (41), 134 (17); Anal. calcd. for $\mathrm{C}_{29} \mathrm{H}_{21} \mathrm{BrN}_{2} \mathrm{O}_{6}$ : C, 60.75; H, 3.69; N, 4.89; found: C, 60.85; H, 3.68; $\mathrm{N}, 4.91 \%$.

Synthesis of 2-bromomethylene-8-ethoxy-2H-chromene-3-carboxylic (methyl-carbonimidic)thioanhydride (16). A solution of compound $4(10 \mathrm{mmol})$ in absolute methanol $(40 \mathrm{~mL})$ was refluxed with potassium thiocyanate $(10 \mathrm{mmol})$ for 2 hours. The solid formed on cooling filtered off, washed with ethanol and dried under vacuum. The product was then recrystallized from acetic acid to give $\mathbf{1 6}$ as brown needles; yield 83\%; m.p. $165-167{ }^{\circ} \mathrm{C}$; IR (KBr) v $\left(\mathrm{cm}^{-1}\right)$ : $3141(\mathrm{NH}), 3093,3021,2985,2890$ (CH stretching); 1717 (CO); ${ }^{1} \mathrm{H}-\mathrm{NMR}\left(\mathrm{DMSO}_{6}\right) \delta: 1.41\left(\mathrm{t}, 3 \mathrm{H}, \mathrm{CH}_{3}, J=7.1 \mathrm{~Hz}\right), 4.10$ (s, 3H, $\left.\mathrm{OCH}_{3}\right), 4.17$ (q, 2H, $\left.\mathrm{CH}_{2}, J=7.1 \mathrm{~Hz}\right), 7.16-7.54(\mathrm{~m}, 4 \mathrm{H}, \mathrm{Ar}-\mathrm{H}, \mathrm{NH}), 7.88(\mathrm{~s}, 1 \mathrm{H},=\mathrm{CH}), 8.58(\mathrm{~s}, 1 \mathrm{H}, \mathrm{H}-4)$; MS m/z (\%): $385\left(\mathrm{M}^{+}+2,37\right), 383\left(\mathrm{M}^{+}, 42\right), 303$ (100), 355 (37), 276 (67), 232 (44), 218 (28); Anal. calcd. for $\mathrm{C}_{15} \mathrm{H}_{14} \mathrm{BrNO}_{4} \mathrm{~S}$ : C, 46.89; H, 3.67; N, 3.65; found: C, 47.01; H, 3.67; N, 3.67\%.

4-Bromo-2-(3-ethoxy-2-hyroxybenzylidine)-3-hydroxybut-3-enoic cyanic thioanhydride (18). A solution of $16(10 \mathrm{mmol})$ was boiled in glacial acetic acid/methanol mixture $(1: 1 ; 40 \mathrm{~mL})$. $\mathrm{HCl}$ gas stream was bubbled into the hot solution for 2 hours. The reaction mixture was allowed to cool down and the solid formed was filtered off and recrystalized from methanol as yellow crystals; yield 89\%; m.p. 235-237 ${ }^{\circ} \mathrm{C}$; Anal. calcd. for $\mathrm{C}_{14} \mathrm{H}_{12} \mathrm{BrNO}_{4} \mathrm{~S}$ (368.97): C, 45.53; H, 3.25; Br, 21.41; N, 3.79; S, 8.67; found: C, 45.55; H, 3.27; Br, 21.42; N, 3.81; S, 8.68\%.

Synthesis of 3-ethoxy-2-hydroxybenzaldehyde azine (19). A solution of compound 16 (5 mmol) in absolute methanol $(20 \mathrm{~mL})$ was stirred at room temperature with $85 \%$ hydrazine hydrate $(10 \mathrm{mmol})$ for 4 hours. The solid obtained filtered, washed with ethanol (five times, $10 \mathrm{~mL}$ each) and dried under reduced pressure. The product was recrystallized from acetic acid to give $\mathbf{1 9}$ as yellow crystals; yield 93\%; m.p. 275-277 ${ }^{\circ} \mathrm{C}$; IR ( $\left.\mathrm{KBr}\right)$ v $\left(\mathrm{cm}^{-1}\right): 3,302(\mathrm{OH}), 3,083,3,025,2,965,2,870$ (CH stretching), 1,562, 1,597 (C=N); ${ }^{1} \mathrm{H}-\mathrm{NMR}\left(\mathrm{DMSO}_{\mathrm{d}}\right) \delta: 1.34\left(\mathrm{t}, 3 \mathrm{H}, \mathrm{CH}_{3}, J=7.0 \mathrm{~Hz}\right), 4.09$ (q, 2H, $\mathrm{CH}_{2}$, $J=7.0 \mathrm{~Hz}), 6.87-7.35(\mathrm{~m}, 6 \mathrm{H}, \mathrm{Ar}-\mathrm{H}), 9.20(\mathrm{~s}, 1 \mathrm{H},=\mathrm{CH}), 12.10,12.45(\mathrm{~s}, 1 \mathrm{H}, \mathrm{OH})$; $\mathrm{MS} \mathrm{m} / z(\%)$ : 
$328\left(\mathrm{M}^{+}, 100\right), 164$ (57), 136 (46), 121 (51), 80 (65); Anal. calcd. for $\mathrm{C}_{18} \mathrm{H}_{20} \mathrm{~N}_{2} \mathrm{O}_{4}$ : C, 65.84; H, 6.14; $\mathrm{N}, 8.53$; found: C, 65.81; H, 6.10; N, 5.55\%.

Synthesis of 3-(2-aminothiazol-4-yl)-8-ethoxycoumarin (20a). Compound 4 (10 mmol) and thiourea $(10 \mathrm{mmol})$ were disolved in absolute methanol $(40 \mathrm{~mL})$. The reaction mixture was refluxed for 1 hour. A precipitate formed on cooling, which was collected by filtration, washed with ethanol and dried under vacuum. The crude product was recrystallized from ethanol to give pale yellow crystals; yield 94\%; m.p. 200-202 ${ }^{\circ} \mathrm{C}$; IR (KBr) v $\left(\mathrm{cm}^{-1}\right)$ : 3,387, 3,302, 3,148 $\left(\mathrm{NH}_{2}\right), 3,020,2,983,2,887(\mathrm{CH}$ stretching), 1,705 (CO); MS m/z (\%): $288\left(\mathrm{M}^{+}, 100\right), 261$ (26), 203 (21), 134 (22), 89 (40); Anal. calcd. for $\mathrm{C}_{14} \mathrm{H}_{12} \mathrm{~N}_{2} \mathrm{O}_{3} \mathrm{~S}$ : C, 58.32; H, 4.20; N, 9.72; found: C, 58.31; H, 4.18; N, 9.73\%.

Synthesis of 3-(2-(phenylamino)thiazol-4-yl)-8-ethoxycoumarin (20b). Compound 20b was prepared from $4(10 \mathrm{mmol})$ and phenylthiourea $(10 \mathrm{mmol})$ according to the procedure described for $\mathbf{2 0 a}$ to give 20b from ethanol/benzene mixture as yellow crystals; yield 69\%; m.p. $216-218^{\circ} \mathrm{C}$; IR $(\mathrm{KBr}) v\left(\mathrm{~cm}^{-1}\right)$ : 3,302, 3,148 (NH), 3,083, 3,011, 2,955, 2,850 (CH stretching), 1,697 (CO); ${ }^{1} \mathrm{H}-\mathrm{NMR}$ (DMSO-d ${ }_{6}$ ) $\delta: 1.41$ (t, 3H, $\mathrm{CH}_{3}, J=7.0 \mathrm{~Hz}$ ), 4.13 (q, 2H, $\left.\mathrm{CH}_{2}, J=7.0 \mathrm{~Hz}\right), 7.00-7.76$ (m, 7H, Ar-H), 8.61 (s, 1H, H-4), 10.31 (s, 1H, HNPh); MS m/z (\%): 364 (M+100), 279 (27), 207 (12), 150 (39), 77 (41); Anal. calcd. for $\mathrm{C}_{20} \mathrm{H}_{16} \mathrm{~N}_{2} \mathrm{O}_{3} \mathrm{~S}$ : C, 65.92; H, 4.43; N, 7.69; found: C, 65.95; H, 4.40; N, 7.71\%.

Synthesis of 2-(4-(8-ethoxycoumarin-3-yl)thiazol-2-yl)acetonitrile hydrobromide (20c). Compound 20c was prepared from $4(10 \mathrm{mmol})$ and cyanothioacetamide $(10 \mathrm{mmol})$ according to the procedure described for 20a to give 20c from benzene as yellow crystals; yield 96\%; m.p. 195-197 ${ }^{\circ} \mathrm{C}$; IR ( $\left.\mathrm{KBr}\right)$ $v\left(\mathrm{~cm}^{-1}\right): 1,722(\mathrm{CO}), 3,021,2,946,2,890\left(\mathrm{CH}\right.$ stretching), 2,262 (CN); ${ }^{1} \mathrm{H}-\mathrm{NMR}\left(\mathrm{DMSO}-\mathrm{d}_{6}\right) \delta: 1.43$ $\left(\mathrm{t}, 3 \mathrm{H}, \mathrm{CH}_{3}, J=7.1 \mathrm{~Hz}\right), 4.16\left(\mathrm{q}, 2 \mathrm{H}, \mathrm{CH}_{2}, J=7.1 \mathrm{~Hz}\right), 4.55\left(\mathrm{~s}, 2 \mathrm{H}, \mathrm{CH}_{2}\right), 7.29-7.32(\mathrm{~m}, 3 \mathrm{H}$,

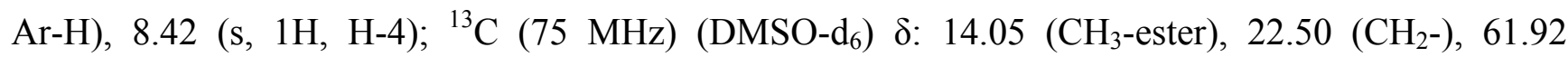
$\left(\mathrm{CH}_{2}\right.$-ester), 115.47 (C-7), $116.43(\mathrm{CN}), 118.95$ (C-5), 122.15 (C-4a), 124.26 (C-5 thiazole), 126.91 (C-6), 127.51 (C-3), 142.11 (C-4 thiazole), 144.92 (C-4), 151.32 (C-8a), 154.54 (C-8), 158.65 (C-2 thiazole), 159.89 (CO-lactone); $\mathrm{MS} \mathrm{m} / z$ (\%): $394\left(\mathrm{M}^{+}+2,24.9\right), 392\left(\mathrm{M}^{+}, 28.9\right), 390364\left(\mathrm{M}^{+}-\mathrm{CO}\right.$, 39.5), $312\left(\mathrm{M}^{+}-\mathrm{HBr}, 54.4\right), 284\left(\mathrm{M}^{+}-(\mathrm{HBr}+\mathrm{CO}), 100\right), 256$ (43.6), 227 (18), 198 (13.7), 134 (11.2), 89 (27.1), 63 (23.1); Anal. calcd. for $\mathrm{C}_{16} \mathrm{H}_{13} \mathrm{BrN}_{2} \mathrm{O}_{3} \mathrm{~S}$ : C, 48.87; H, 3.33; N, 7.12; found: C, 49.25; H, 2.84; N, 7.20\%.

Synthesis of 2-(4-(8-ethoxycoumarin-3-yl)thiazol-2-yl)-3-ethoxyacrylonitrile hydrobromide (21). A solution of compound 20c $(5 \mathrm{mmol})$ was refluxed with triethyl orthoformate $(5 \mathrm{~mL})$ for 3 hours. The excess triethyl orthoformate removed under reduced pressure. The residual solid was treated with ethanol, filtered off, washed with ethanol (three times, $10 \mathrm{~mL}$ each) and dried under vacuum. The crude product was recrystallized from benzene/petroleum ether $40-60{ }^{\circ} \mathrm{C}$ mixture to give compound 21 as green crystals; yield 83\%; m.p. $145-147{ }^{\circ} \mathrm{C}$; IR $(\mathrm{KBr})$ v $\left(\mathrm{cm}^{-1}\right): 3,027,2,966,2,876(\mathrm{CH}$ stretching), 2,222 (CN), 1,728 (CO); ${ }^{1} \mathrm{H}-\mathrm{NMR}\left(\mathrm{DMSO}_{\mathrm{d}}\right) \delta: 1.39\left(\mathrm{t}, 3 \mathrm{H}, \mathrm{CH}_{3}, J=6.9 \mathrm{~Hz}\right), 1.42$ (t, $\left.3 \mathrm{H}, \mathrm{CH}_{3}, J=7.2 \mathrm{~Hz}\right), 4.17\left(\mathrm{q}, 2 \mathrm{H}, \mathrm{CH}_{2}, J=6.9 \mathrm{~Hz}\right), 4.45\left(\mathrm{q}, 2 \mathrm{H}, \mathrm{CH}_{2}, J=7.2 \mathrm{~Hz}\right), 7.28-7.45(\mathrm{~m}, 3 \mathrm{H}$, $\operatorname{Ar}-\mathrm{H}), 8.15(\mathrm{~s}, 1 \mathrm{H},=\mathrm{CH}), 8.43(\mathrm{~s}, 1 \mathrm{H}, 4-\mathrm{H}) ; \mathrm{MS} \mathrm{m} / z(\%): 450\left(\mathrm{M}^{+}+2,10.1\right), 448\left(\mathrm{M}^{+}, 12.1\right)$, 368 (100), 340 (50.1), 312 (67), 227 (29.3), 134 (35.7), 89 (49.9); Anal. calcd. for $\mathrm{C}_{19} \mathrm{H}_{17} \mathrm{BrN}_{2} \mathrm{O}_{4} \mathrm{~S}$ : C, 50.79; H, 3.81; N, 6.23; found: C, 51.14; H, 3.35; N, 6.30\%. 
Synthesis of 2-(4-(8-ethoxycoumarin-3-yl)thiazol-2-yl)-3-(dimethylamino)acrylonitrile hydrobromide (22). A mixture of $21(2.5 \mathrm{mmol})$ and dimethylamine $(2.5 \mathrm{mmol})$ in absolute methanol $(30 \mathrm{~mL})$ was refluxed for 6 hours. The resulting solid was filtered off, washed with ethanol and dried. The crude product was then crystallized from acetic acid to give compound 22 as brown crystals; yield 89\%; m.p. 206-208 ${ }^{\circ} \mathrm{C}$; IR (KBr) v $\left(\mathrm{cm}^{-1}\right)$ : 3,012, 2,944, 2,927, 2,890, 2,874 (CH stretching), 2,191 (CN), 1,720 (CO); ${ }^{1} \mathrm{H}-\mathrm{NMR}\left(\mathrm{DMSO}_{6}\right) \delta: 1.41\left(\mathrm{t}, 3 \mathrm{H}, \mathrm{CH}_{3}, J=6.9 \mathrm{~Hz}\right), 3.28\left(\mathrm{~s}, 6 \mathrm{H},\left(\mathrm{CH}_{3}\right)_{2}\right), 4.18\left(\mathrm{q}, 2 \mathrm{H}, \mathrm{CH}_{2}\right.$, $J=6.9 \mathrm{~Hz}), 7.22-7.59$ (m, 3H, Ar-H), 7.89 (s, 1H, C=CHNMe $), 8.12(\mathrm{~s}, 1 \mathrm{H}, 4-\mathrm{H})$; MS m/z (\%); $449\left(\mathrm{M}^{+}+2\right.$, 14.1), $447\left(\mathrm{M}^{+}, 18\right), 367\left(\mathrm{M}^{+}-\mathrm{HBr}, 100\right), 334$ (35.8), 230 (28.6), 170 (18.3), 132 (29.1), 89 (48.9); Anal. calcd. for $\mathrm{C}_{19} \mathrm{H}_{18} \mathrm{BrN}_{3} \mathrm{O}_{3} \mathrm{~S}$ : C, 50.90; $\mathrm{H}, 4.05 ; \mathrm{N}, 9.37$; found: $\mathrm{C}, 51.24 ; \mathrm{H}, 3.61$; $\mathrm{N}, 9.46 \%$.

Synthesis of 3-(2-(coumarin-3-yl)thiazol-4-yl)-8-ethoxycoumarin hydrobromide (24). A solution of the acetonitrile derivative 20 c $(10 \mathrm{mmol})$ in absolute methanol $(40 \mathrm{~mL})$ was refluxed with salicyaldehyde (10 mmol) in the presence of piperidine for 2 hours. The solid formed was filtered off, washed with ethanol and dried under reduced pressure. The crude product was recrystallized from acetic acid to give compound 24 as pale yellow crystals; yield 91\%; m.p. $265-7{ }^{\circ} \mathrm{C}$; IR $(\mathrm{KBr}) v\left(\mathrm{~cm}^{-1}\right): 2,948,2,927$, 2,897, 2,874 (CH stretching), 1,720 (CO); ${ }^{1} \mathrm{H}-\mathrm{NMR}$ (DMSO-d 6 ) $\delta: 1.43$ (t, 3H, $\mathrm{CH}_{3}, J=7.0 \mathrm{~Hz}$ ), 4.19 (q, 2H, $\mathrm{CH}_{2}, J=7.0 \mathrm{~Hz}$ ), 7.31-8.00 (m, 7H, Ar-H), 8.54, 8.95 (s, 1H, H-4), 9.14 (s, 1H, thiazole-H); Anal. calcd. for $\mathrm{C}_{23} \mathrm{H}_{16} \mathrm{BrNO}_{5} \mathrm{~S}: \mathrm{C}, 55.34 ; \mathrm{H}, 3.24 ; \mathrm{N}, 2.81$; found: $\mathrm{C}, 55.77 ; \mathrm{H}, 2.85 ; \mathrm{N}, 2.84 \%$.

Synthesis of 3-(2-(2-imino-6,8-diiodo-coumarin-3-yl)thiazol-4-yl)-8-ethoxycoumarine hydrobromide (25). Compound 25 was prepared from 20c $(10 \mathrm{mmol})$ and 3,5-diiodosalicyaldehyde $(10 \mathrm{mmol})$ according to the procedure described for $\mathbf{2 4}$ to give $\mathbf{2 5}$ from benzene as pale green crystals; yield 96\%; m.p. 310-312 ${ }^{\circ} \mathrm{C}$; IR (KBr) v $\left(\mathrm{cm}^{-1}\right): 2,938,2,927,2,898,2,877$ (CH stretching), 1,720 (CO); MS m/z (\%): $749\left(\mathrm{M}^{+}, 15.9\right), 669$ (100), 668 (59.6), 641 (69), 613 (24), 585 (14), 321 (13), 190 (15), 134 (14), 89 (19). Anal. calcd. for $\mathrm{C}_{23} \mathrm{H}_{14} \mathrm{BrI}_{2} \mathrm{NO}_{5} \mathrm{~S}: \mathrm{C}, 36.83 ; \mathrm{H}, 1.88 ; \mathrm{N}, 1.87$; found: C, 36.98; H, 1.63; $\mathrm{N}, 1.89 \%$.

Synthesis of 3-(2-(6-(dimethylamino)-2-iminocoumarin-3-yl)thiazol-4-yl)-8-ethoxycoumarin hydrobromide (26). Compound 26 was prepared from 20c $(10 \mathrm{mmol})$ and 5-dimethylaminosalicyaldehyde (10 mmol) according to the procedure described for $\mathbf{2 4}$ to give $\mathbf{2 6}$ from benzene as brown crystals; yield: $90 \%$ m.p. 223-5 ${ }^{\circ} \mathrm{C}$; IR $(\mathrm{KBr})$ v $\left(\mathrm{cm}^{-1}\right)$ : 3,449, 3,209, (NH), 3,015, 2,978, 2,921, 2,890, 2,873 (CH stretching), 1,597 (C=N), 1,720 (CO); ${ }^{1} \mathrm{H}-\mathrm{NMR}\left(\mathrm{DMSO}_{-} \mathrm{d}_{6}\right) \delta: 1.43\left(\mathrm{t}, 3 \mathrm{H}, \mathrm{CH}_{3}, J=7.1 \mathrm{~Hz}\right), 3.31$ (s, $\left.6 \mathrm{H}, 2 \mathrm{CH}_{3}\right), 4.19$ (q, 2H, $\left.\mathrm{CH}_{2}, J=7.1 \mathrm{~Hz}\right), 6.63-7.67$ (m, 7H, Ar-H and thiazole-H), 8.39 (s, 2H, H-4); Anal. calcd. for $\mathrm{C}_{25} \mathrm{H}_{22} \mathrm{BrN}_{3} \mathrm{O}_{4} \mathrm{~S}$ : C, 55.56; H, 4.10; N, 7.78; found: C, 55.88; H, 3.74; N, 7.83\%.

\subsection{Reaction of 20c with Aromatic Aldehydes}

A solution of 20c $(5 \mathrm{mmol})$ in absolute methanol $(40 \mathrm{~mL})$ was refluxed with 4-methoxybenzaldehyde and/or 4-chlorobenzaldehyde $(5 \mathrm{mmol})$ in the presence of piperidine for 1 hour. The solid formed was filtered off, washed with ethanol and dried under reduced pressure. The crude product was recrystallized from a suitable solvent to give $\mathbf{2 7 a}, \mathbf{b}$. The physical and spectral data

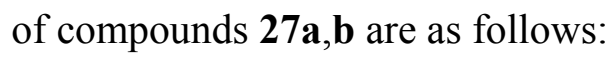


3-(4-Methoxyphenyl)-2-(4-(8-ethoxycoumarin-3-yl)thiazol-2-yl)acrylonitrile hydrobromide (27a). Yellow crystals; yield 91\%; m.p. 215-7 ${ }^{\circ} \mathrm{C}$; IR $(\mathrm{KBr}) v\left(\mathrm{~cm}^{-1}\right): 3,425,3,139,2,923$ (NH and $\mathrm{CH}$ stretching), 2,207 (CN), 1,728 (CO); MS m/z (\%): 510 [M+] (7.5), 508 (76.3), 430 (100), 265 (15), 216 (42), 134 (15), 89 (34); Anal. calcd. for $\mathrm{C}_{24} \mathrm{H}_{19} \mathrm{BrN}_{2} \mathrm{O}_{4} \mathrm{~S}: \mathrm{C}, 56.37 ; \mathrm{H}, 3.74 ; \mathrm{N}, 5.48$; found: $\mathrm{C}, 56.71 ; \mathrm{H}, 3.37 ; \mathrm{N}, 5.53 \%$.

3-(4-Chlorophenyl)-2-(5-bromo-4-(8-ethoxycoumarin-3-yl)thiazol-2-yl)acrylonitrile hydrobromide (27b). Yellow crystals; yield 86\%; m.p. 196-7 ${ }^{\circ} \mathrm{C}$; IR $(\mathrm{KBr}) v\left(\mathrm{~cm}^{-1}\right): 3,011,3,332.8$ and 3,224.8, 2,923 (NHBr and $\mathrm{CH}$ stretching), 2,214 (CN), 1,720 (CO); $\mathrm{MS} m / z(\%): 516\left(\mathrm{M}^{+}+4,31.5\right), 514\left(\mathrm{M}^{+}+2,100\right)$, $512\left(\mathrm{M}^{+}, 34.9\right), 433$ (76.5), 295 (46), 215 (63), 125 (57), 89 (69); Anal. calcd. for $\mathrm{C}_{23} \mathrm{H}_{16} \mathrm{BrClN}_{2} \mathrm{O}_{3} \mathrm{~S}$ : C, 53.56; H, 3.13; N, 5.43; found: C, 53.90; H, 2.71; N, 5.48\%.

Synthesis of 2-(2-(4-methoxyphenyl)hydrazono)-2-(4-(8-ethoxycoumarin-3-yl)thiazol-2-yl)acetonitrile hydrobromide (28). Method A: To a solution of compound $20 \mathrm{c}(10 \mathrm{mmol})$ in methanol $(20 \mathrm{~mL})$, a combination of 4-methoxyaniline $(10 \mathrm{mmol})$, hydrochloric acid $(5 \mathrm{~mL})$ and sodium nitrite $(10 \mathrm{mmol})$ in glacial acetic acid $(20 \mathrm{~mL})$ at $0{ }^{\circ} \mathrm{C}$ was added dropwise. The resulting mixture was stirred at $0{ }^{\circ} \mathrm{C}$ for 1 hour then the stirring was continued for 2 hours more at room temperature. The solid formed was filtered, washed with ethanol and dried under vacuum. The crude product was recrystallized from dioxane to give pale green crystals; yield 73\%; m.p. $258-60{ }^{\circ} \mathrm{C}$; IR $(\mathrm{KBr}) v\left(\mathrm{~cm}^{-1}\right): 3,157(\mathrm{NH}), 3,011$, 2,883 (CH stretching), 2,214 (CN), 1,735.8 (CO); ${ }^{1} \mathrm{H}-\mathrm{NMR}\left(\mathrm{DMSO}_{6}\right)$ 8: $1.44\left(\mathrm{t}, 3 \mathrm{H}, \mathrm{CH}_{3}\right.$, $J=6.9 \mathrm{~Hz}), 3.76\left(\mathrm{~s}, 3 \mathrm{H}, \mathrm{OCH}_{3}\right), 4.18\left(\mathrm{q}, 2 \mathrm{H}, \mathrm{CH}_{2}, J=6.9 \mathrm{~Hz}\right), 6.96-7.47$ (m, 7H, Ar-H), 8.39 (s, 1H, $\mathrm{H}-4), 11.93$ (s, 1H, NH, cancelled by $\left.\mathrm{D}_{2} \mathrm{O}\right)$; $\mathrm{MS} m / z(\%): 526\left(\mathrm{M}^{+}, 12\right), 445(100)$. Anal. calcd. for $\mathrm{C}_{23} \mathrm{H}_{19} \mathrm{BrN}_{4} \mathrm{O}_{4} \mathrm{~S}$ : C, 52.38; H, 3.63; N, 10.62; found: C, 52.69; H, 3.26; N, 10.69\%. Method B: A solution of $4(5 \mathrm{mmol})$ in absolute methanol $(20 \mathrm{~mL})$ was refluxed with (4-methoxyphenylazo)-2cyanoethanethioamide $29(5 \mathrm{mmol})$. The work up continued as previously mentioned in Method A to give 28; yield $79 \%$; (identified by m.p. and mixed m.p.).

\subsection{Antibacterial Activity}

The newly synthesized compounds were screened for their antimicrobial activities in vitro against two Gram-negative Bordetella bronchiseptica (ATCC 4617) and Escherichia coli (ATCC 14169) and four Gram-positive Bacillus pumilus (ATCC 14884), Bacillus subtilis (ATCC 6633), Staphylococcus aureus (ATCC 29737) and Staphylococcus epidermidis (ATCC 12228) pathogenic bacteria and two fungi Candida albicans (ATCC 10231) and Saccharomyces cervesia (ATCC 9080). The activities of these compounds were tested using the disc diffusion method [40] for bacteria and the paper disk diffusion method [41] for fungi. The area of zone of inhibition was measured using Ampicillin $\left(25 \mu \mathrm{g} \mathrm{mL}^{-1}\right)$ as standard antibiotic Micostatin $\left(25 \mu \mathrm{g} \mathrm{mL}^{-1}\right)$ was used as a reference antifungal. The tested compounds were dissolved in $N, N$-dimethylformamide (DMF) to give a solution of $1 \mathrm{mg} \mathrm{mL}{ }^{-1}$. The inhibition zones were measured in millimeters at the end of an incubation period of 48 hours at $28{ }^{\circ} \mathrm{C}$. $N, N$-dimethylformamide (DMF) showed no inhibition zone. Test results are shown in Table 1. 
Table 1. Antibacterial screening of the synthetic compounds.

\begin{tabular}{|c|c|c|c|c|c|c|c|c|}
\hline \multirow[b]{2}{*}{$\begin{array}{c}\text { Compd. } \\
\text { No. }^{\mathrm{a}}\end{array}$} & \multicolumn{8}{|c|}{ Inhibition Zone Diameter in mm } \\
\hline & $\begin{array}{c}\text { Bordetella } \\
\text { bronchiseptica } \\
\text { ATCC } \\
4617 \\
\end{array}$ & $\begin{array}{c}\text { Escheri- } \\
\text { chia coli } \\
\text { ATCC } \\
14169\end{array}$ & $\begin{array}{c}\text { Bacillus } \\
\text { pumilus } \\
\text { ATCC } \\
14884 \\
\end{array}$ & $\begin{array}{c}\text { Bacillus } \\
\text { Subtilis } \\
\text { ATCC } \\
6633 \\
\end{array}$ & $\begin{array}{l}\text { Staph. } \\
\text { aureus } \\
\text { ATCC } \\
29737 \\
\end{array}$ & $\begin{array}{c}\text { Staph. } \\
\text { Epidermidis } \\
\text { ATCC } \\
12228 \\
\end{array}$ & $\begin{array}{c}\text { Candida } \\
\text { albicans } \\
\text { ATCC } \\
10231 \\
\end{array}$ & $\begin{array}{c}\text { Saccharomyces } \\
\text { cerevisae } \\
\text { ATCC } \\
9080 \\
\end{array}$ \\
\hline 3 & 19 & 20 & 11 & 12 & 19 & 12 & 18 & 10 \\
\hline 4 & 22 & 27 & 23 & 20 & 20 & 16 & 13 & 15 \\
\hline 5 & 24 & 18 & 28 & 28 & 27 & 25 & 15 & 21 \\
\hline 6 & 28 & 14 & 18 & 26 & 15 & 18 & 24 & 18 \\
\hline 7 & 22 & 13 & 19 & 23 & 14 & 13 & 17 & 22 \\
\hline 8 & NA & NA & NA & NA & NA & NA & NA & NA \\
\hline 9 & NA & 13 & NA & NA & 10 & NA & NA & NA \\
\hline 11 & NA & NA & NA & NA & NA & NA & NA & NA \\
\hline 12 & NA & 14 & NA & NA & 14 & NA & NA & NA \\
\hline 16 & 27 & 19 & 18 & 24 & 12 & 13 & 17 & 13 \\
\hline $20 a$ & NA & 10 & NA & NA & 10 & NA & NA & NA \\
\hline $20 b$ & NA & 11 & NA & NA & 12 & NA & NA & NA \\
\hline $20 \mathrm{c}$ & 13 & 10 & 18 & 11 & 11 & 20 & 14 & 19 \\
\hline 21 & 22 & 10 & 18 & 20 & 11 & 19 & 22 & 11 \\
\hline 22 & 20 & 11 & 20 & 19 & 13 & 9 & 13 & 15 \\
\hline 24 & 13 & 10 & NA & 21 & 10 & NA & NA & 12 \\
\hline 25 & 8 & 14 & NA & 14 & 15 & NA & NA & 14 \\
\hline $27 \mathbf{a}$ & 19 & 10 & 14 & 18 & 11 & 20 & 16 & 19 \\
\hline $27 \mathrm{~b}$ & 20 & 10 & 14 & 21 & 11 & 13 & 19 & 11 \\
\hline 28 & 27 & 10 & 22 & 22 & 11 & 19 & 23 & 20 \\
\hline Ampicillin* & 24 & 25 & 20 & 25 & 26 & 25 & - & - \\
\hline Mycostatin* & - & - & - & - & - & - & 22 & 24 \\
\hline
\end{tabular}

$\mathrm{NA}=$ not active; Diameter of the hole $=10 \mathrm{~mm} ;{ }^{*} 25 \mu \mathrm{g} / \mathrm{mL},{ }^{\mathrm{a}} \mathrm{c}=1 \mathrm{mg} / \mathrm{mL}$ of new compounds in DMF.

\section{Conclusions}

Our interest in the synthesis of the title compounds was to focus on their study as antimicrobial agents as a part of our program which is aimed at the development of new heterocyclic compounds as more potent antimicrobial agents. In this paper we reported the synthesis of some 8-ethoxycoumarin derivatives bearing side chains, thiazole derivatives and the antimicrobial evaluation of all the novel compounds. The structures of the novel compounds were elucidated on the basis of IR, ${ }^{1} \mathrm{H}-\mathrm{NMR}$, ${ }^{13} \mathrm{C}-\mathrm{NMR}$ and MS data. The screening results demonstrated that replacing the hydrogen atom attached to the coumarin nucleus at C-3 with a side chain as in compound $\mathbf{5}$ and thiazoles $\mathbf{7}$ and $\mathbf{2 8}$ results in wide spectrum antimicrobial activity against all tested bacteria and fungi compared to ampicillin and mycostatin, while the other compounds with other side chains showed moderate to weak activity.

\section{Conflict of Interest}

The authors declare no conflict of interest. 


\section{References and Notes}

1. Kennedy, R.O.; Thornes, R.D. Coumarins: Biology, Applications and Mode of Action; Wiley \& Sons: Chichester, UK, 1997.

2. Murray, R.D.H.; Mendez, J.; Brown, S.A. The Natureal Coumarins; Occurrence, Chemistry and Biochemistry; Wiley \& Sons: New York, NY, USA, 1982.

3. Egan, D.; Kennedy, R.O.; Moran, E.; Cox, D.; Prosser, E.; Thornes, R.D. The pharmacology, metabolism, analysis, and applications of coumarin and coumarin-related compounds. Drug Metab. Rev. 1990, 22, 503-529.

4. Gabor, M. The Pharmacology of Benzopyran Derivatives and Related Compounds; Akademiai Kiado: Budapest, Hungary, 1988; pp. 91-126.

5. Guilbault, G.G.; Practical Fluorescence. Theory, Methods and Techniques; Marcel Dekker: New York, NY, USA, 1973; p. 354.

6. Rathbone, D.L.; Wang, D.; Su, Y.; Billington, D.C. Molecular recognition by fluorescent imprinted polymers. Tetrahedron Lett. 2000, 41, 123-126.

7. Abyshev, A.Z.; Gindin, V.A.; Semenov, E.V.; Agaev, E.M.; Abdulla-zade, A.A.; Guseinov, A.B. Structure and biological properties of 2H-1-benzopyran-2-one (coumarin) derivatives. Pharm. Chem. J. 2006, 40, 607-610.

8. Al-Bayati, R.I.; Hussain Al-Amiery, A.A.; Al-Majedy, Y.K. Design, synthesis and bioassay of novel coumarins. Afr. J. Pure Appl. Chem. 2010, 4, 74-86.

9. Lee, S.; Sivakumar, K.; Shin, W.S.; Xie, F.; Wang, Q. Synthesis and anti-angiogenesis activity of coumarin derivatives. Bioorg. Med. Chem. Lett. 2006, 16, 4596-4599.

10. Monteiro, V.F.F.; Mathias, L.; Vieira, I.J.C.; Schripsema, J.; Braz-Filho, R. Prenylated coumarins, chalcone and new cinnamic acid and dihydrocinnamic acid derivatives from brosimum gaudichaudii. J. Braz. Chem. Soc. 2002, 13, 281-287.

11. Satyanarayana, V.S.V.; Sreevani, P.; Sivakumar, A.; Vijayakumar, V. Synthesis and antimicrobial activity of new Schiff bases containing coumarin moiety and their spectral characterization. ARKIVOC 2008, xvii, 221-233.

12. Nofal, Z.M.; El-Zahar, M.I.; Abd El-Karim, S.S. Novel coumarin derivatives with expected biological activity. Molecules 2000, 5, 99-113.

13. Radanyi, C.; Bras, G.L.; Messaoudi, S.; Bouclier, C.; Peyrat, J.F.; Brion, J.D.; Marsaud, V.; Renoir, J.M.; Alami, M. Synthesis and biological activity of simplified denoviose-coumarins related to novobiocin as potent inhibitors of heat-shock protein 90 (hsp90). Bioorg. Med. Chem. Lett. 2008, 18, 2495-2498.

14. Ahmad, R.; Asad, M.; Siddiqui, Z.N.; Kumar, A. Screening of synthetic new heterocyclic derivatives of 3-formyl-4-hydroxycoumarin for anti-inflammatory activity in albino rats. J. Pharm. Res. Health Care 2009, 1, 46-62.

15. Kostova, I. Synthetic and natural coumarins as cytotoxic agents. Curr. Med. Chem. Anticancer Agents 2005, 5, 29-46.

16. El-Agrody, A.M. Condensation Reactions of $\alpha$-Cyanocinnamonitriles with Naphthols: Synthesis of Naphthopyranopyrimidines and a Naphthopyranone. J. Chem. Res. Synopses 1994, 280-281. 
17. El-Agrody, A.M.; Emam, H.A.; El-Hakim, M.H.; Abd El-Latif, M.S.; Fakery, A.H. Activated nitriles in heterocyclic synthesis: Synthesis of Pyrano[2,3- $d$ ]pyrimidine and Pyrano[3,2-e][1,2,4]triazolo[1,5-c]pyrimidine derivatives. J. Chem. Res. Synopses 1997, 320-321.

18. Bedair, A.H.; El-Hady, N.A.; Abd El-Latif, M.S.; Fakery, A.H.; El-Agrody, A.M. 4-Hydroxycoumarin in heterocyclic synthesis-Part III. Synthesis of some new pyrano[2,3-d] pyrimidine, 2-substituted $[1,2,4]$ triazolo[1,5-c]pyrimidine and pyrimido[1,6- $b][1,2,4]$ triazine derivatives. Farmaco 2000, 55, 708-714.

19. El-Agrody, A.M.; El-Hakim, M.H.; Abd El-Latif, M.S.; Fakery, A.H.; El-Sayed, E.S.M.; El-Ghareab, K.A. Synthesis of pyrano[2,3- $d]$ pyrimidine and pyrano[3,2-e]-[1,2,4]triazolo [2,3-c]pyrimidine derivatives with promising antibacterial activity. Acta Pharm. 2000, 50, 111-120.

20. Sayed, A.Z.; El-Hady, N.A.; El-Agrody, A.M. Condensation of $\alpha$-cyanocinnamonitriles with 6-bromo-2-naphthol: synthesis of pyrano[2,3- $d]$ pyrimidine and pyrano[3,2-e][1,2,4]triazolo [2,3-c]pyrimidine derivatives. J. Chem. Res. Synopses 2000, 164-166.

21. El-Agrody, A.M.; Abd El-Latif, M.S.; El-Hady, N.A.; Fakery, A.H.; Bedair, A.H. Heteroaromatization with 4-Hydroxycoumarin Part II: Synthesis of Some New Pyrano[2,3-d] pyrimidines, $[1,2,4]$ triazolo[1,5-c]pyrimidines and Pyrimido[1,6-b][1,2,4]triazine Derivatives. Molecules 2001, 6, 519-527.

22. Bedair, A.H.; Emam, H.A.; El-Hady, N.A.; Ahmed, K.A.R.; El-Agrody, A.M. Synthesis and antimicrobial activities of novel naphtho[2,1-b]pyran, pyrano[2,3- $d]$ pyrimidine and pyrano[3,2-e] [1,2,4]triazolo[2,3-c]-pyrimidine derivatives. II Farmaco 2001, 56, 965-973.

23. El-Agrody, A.M.; Eid, F.A.; Emam, H.A.; Mohamed, H.M.; Bedair, A.H. Synthesis of 9-Methoxy and 9-Acetoxy-3-amino-1-(4-methoxyphenyl)-1H-benzo[f]chromene-2-carbonitriles via 2-(iminopiperidin-1-yl-methyl)-3-(4-methoxyphenyl)acrylonitrile as Intermediate. Z. Naturforsch. B 2002, 57b, 579-585.

24. Khafagy, M.M.; Abd El-Wahab, A.H.F.; Eid, F.A.; El-Agrody, A.M. Synthesis of halogen derivatives of benzo $[h]$ chromene and benzo $[a]$ anthracene with promising antimicrobial activities. Farmaco 2002, 57, 715-722.

25. Eid, F.A.; Bedair, A.H.; Emam, H.A.; Mohamed, H.M.; El-Agrody, A.M. Reaction of activated nitriles with methanolic piperidine and synthesis of $1 H$-Benzo[f]chromene, Diazabenzo[j]anthracene and Diazabenzo[a]- $[1,2,4]$ triazolo[j]anthracene derivatives. Al-Azhar Bull. Sci. 2003, 14, 311-327.

26. Abd-El-Aziz, A.S.; El-Agrody, A.M.; Bedair, A.H.; Christopher Corkery, T.; Ata, A. Synthesis of hyroxyquinoline derivatives, aminoheydroxychromene, aminocoumarin and their anitimicrobial activities. Heterocycles 2004, 63, 1793-1812.

27. Abd-El-Aziz, A.S.; Mohamed, H.M.; Mohammed, S.; Zahid, S.; Ata, A.; Bedair, A.H.; El-Agrody, A.M.; Harvey, P.D. Synthesis of novel coumarin and benzocoumarin derivatives and their biological and photophysical studies. J. Heterocycl. Chem. 2007, 44, 1287-1301.

28. Sabry, N.M.; Mohamed, H.M.; Motlaq, S.S.; El-Agrody, A.M. Synthesis of $4 H$-chromene, coumarin, $12 H$-chromeno[2,3-d]pyrimidine derivatives and some of their antimicrobial and cytotoxicity activities. Eur. J. Med. Chem. 2011, 46, 765-772. 
29. Zhuravel, I.O.; Kovalenko, S.M.; Vlasov, S.V.; Chernykh, V.P. Solution phase synthesis of a combinatorial library of 3-[4-(coumarins-3-yl)-1,3-thiazol-2-yl carbamoyl]propanoic acid amides. Molecules 2005, 10, 444-456.

30. Czerney, P.; Jena, V.C.Z.; Hartmann, H. Heterocyclic substituted coumarins from $\beta$-chloropropeniminium salts. J. Prakt. Chem. 1982, 324, 255-266.

31. Koelsch, C.F.; Embree, H.D. Condensation of 3-acetylcoumarin with acetone and amines. J. Org. Chem. 1958, 23, 1606-1608.

32. O'Callaghan, C.N.; McMurry, T.B.H. Dimerisation of Kneovenagel condensation products obtained from simple unconjugated and $\alpha, \beta$-unsaturated ketones. J. Chem. Res. Synopses 1999, 458-459.

33. Zhou, J.F.; Gong, G.X.; An, L.T.; Liu, Y.; Zhu, F.X.; Zhu, Y.L.; Ji, S.J. An efficient synthesis of quinoxalines under catalyst free and microwave-irradiation conditions. Synlett 2008, 20, 3163-3166.

34. Kumar, P.V.; Rao, V.R. Synthesis and biological activity of some 3-imidazo [1,2-a] pyridin2-yl-chromen-2-one and 3-indolizin-2-yl-chromen-2-one. Indian J. Chem. Sect. B 2005, 44, 2120-2125.

35. Ramanna, S.; Rao, V.R.; Kumari, T.S.; Rao, T.V.P. Synthesis of N-(4-2H-1-benzopyran-2-one)2-thiazolyl)phthalimides. Phosphorous Sulfur Silicon 1995, 107, 197-204.

36. Islam, A.M.; Ali, F.M.; El-Sharief, A.M.Sh.; Bedair, A.H.; El-Masry, F.M. Some reactions of coumarins with hydrazine and ethylenediamine. Egypt J. Chem. 1983, 26, 233-239.

37. Chunduru, R.; Sreenivasa, V.; Vedula, R.R. One pot synthesis of 3-[2-(arylamino)-thiazole-4yl]coumarins in a three-component synthesis and a catalyst and solvent-free synthesis grinding. J. Chem. Res. 2010, 34, 50-53.

38. Santhosh, P.; Chunduru, V.S.R.; Rao, V.R. One pot synthesis of tri-substituted pyrazoles via multi-component approach. Chem. Heterocycl. Comp. 2011, 47, 448-451.

39. Czerney, P.; Jena, V.C.Z.; Hartmann, H. 3- $\alpha$-Bromoacetylcoumarines as synthones for heterocyclic substituted coumarins. J. Prakt. Chem. 1983, 325, 551-560.

40. European Committee for Antimicrobial Susceptibility Testing (EUCAST) of the European Society of Clinical Microbiology and Infectious Diseases (ESCMID). Determination of minimum inhibitory concentrations (MICs) of antibacterial agents by agar dilution. Clin. Microbiol. Infect. 2000, 6, 509-515.

41. National Committee for Clinical Laboratory Standards (NCCLS). Methods for Dilution Antimicrobial Susceptibility Tests for Bacteria that Grow Aerobically, 5th ed.; Approved Standard M7-A5. NCCLS: Wayne, PA, USA, 2000.

Sample Availability: Samples of the all compounds are available from the authors.

(C) 2012 by the authors; licensee MDPI, Basel, Switzerland. This article is an open access article distributed under the terms and conditions of the Creative Commons Attribution license (http://creativecommons.org/licenses/by/3.0/). 